MATHEMATICS OF COMPUTATION

Volume 73, Number 245, Pages 211-242

S 0025-5718(03)01535-7

Article electronically published on April 22, 2003

\title{
LAGUERRE APPROXIMATION OF STABLE MANIFOLDS WITH APPLICATION TO CONNECTING ORBITS
}

\author{
GERALD MOORE
}

\begin{abstract}
We present an algorithm, based on approximation by Laguerre polynomials, for computing a point on the stable manifold of a stationary solution of an autonomous system. A superconvergence phenomenon means that the accuracy of our results is much higher than the usual spectral accuracy. Both the theory and the implementation of the method are considered. Finally, as an application of the algorithm, we describe a fully spectral approximation of homo- and heteroclinic orbits.
\end{abstract}

\section{INTRODUCTION}

We consider an autonomous system

$$
\mathbf{v}^{\prime}=\mathbf{F}(\mathbf{v}), \quad \mathbf{F}: \mathbb{R}^{m} \mapsto \mathbb{R}^{m},
$$

possessing a stationary solution, which without loss of generality we assume to be the origin; i.e., $\mathbf{F}(\mathbf{0})=\mathbf{0}$. Our aim in this paper is to introduce a new algorithm, particularly suitable for smooth $\mathbf{F}$, for approximating a point on the local stable manifold of zero for (1.1). For the rest of this introduction, we briefly describe the elementary properties of this manifold which will be required; for more details see [14], [21], [24].

We assume that $\mathbf{F}$ is differentiable at the origin and additionally that

$$
\mathbf{F}(\mathbf{x}) \equiv \mathbf{A x}+\mathbf{G}(\mathbf{x})
$$

where A denotes the $m \times m$ Jacobian matrix and $\|\mathbf{G}(\mathbf{x})\|=O\left(\|\mathbf{x}\|^{2}\right)$. Note that $\|\cdot\|$ will always denote the vector 2-norm and that we shall be more specific about the structure of $\mathbf{G}$ later. In this paper we shall also assume that the origin is hyperbolic, i.e., that A has no zero or purely imaginary eigenvalues, although an extension to the general case is described in section 6 . We make frequent use of the decomposition

$$
\mathbb{R}^{m}=\mathcal{E}_{s} \oplus \mathcal{E}_{u},
$$

where the stable subspace $\mathcal{E}_{s}$ is the invariant subspace of A corresponding to those eigenvalues with strictly negative real part, while the unstable subspace $\mathcal{E}_{u}$ is the invariant subspace of $A$ corresponding to those eigenvalues with strictly positive real part. These subspaces have dimensions $p^{s} \& p^{u} \equiv m-p^{s}$, respectively, and we use $P^{s} \& P^{u}$ to denote the projections induced by the above direct sum. The local

Received by the editor February 20, 2001 and, in revised form, May 13, 2002.

2000 Mathematics Subject Classification. Primary 33C45, 37C29, 37M99, 65N35, 65P40.

Key words and phrases. Laguerre polynomials, invariant manifolds, homoclinic orbits, spectral methods. 
stable manifold is $p^{s}$-dimensional and consists of those points lying on orbits which converge to the origin as $t \rightarrow \infty$. It may be parametrised by $\mathcal{E}_{s}$ near the origin and in fact this subspace is tangent to the manifold at the origin. Hence, given $\boldsymbol{\xi} \in \mathcal{E}_{s}$ sufficiently small, the unique point $\mathbf{y}$ on the manifold satisfying $P^{s} \mathbf{y}=\boldsymbol{\xi}$ is obtained by solving (1.1) with boundary conditions

$$
P^{s} \mathbf{v}(0)=\boldsymbol{\xi} \quad \text { and } \quad \lim _{t \rightarrow \infty} P^{u} \mathbf{v}(t)=\mathbf{0} .
$$

If we denote this solution by $\mathbf{v}^{\star}(t)$, the effect of (1.4) is to force $\mathbf{v}^{\star}(t)$ to converge exponentially to $\mathbf{0}$ as $t \rightarrow \infty$ and so $\mathbf{y}$ is given by $\mathbf{v}^{\star}(0)$.

The contents of this paper are as follows. In section 2 we construct the Galerkin (and equivalent collocation and Laguerre expansion) equations approximating (1.1) and (1.4). In section 3 we prove that these discrete equations have a unique local solution and provide a superconvergent approximation to the stable manifold. In section 4 we describe how to implement our algorithm efficiently and then, in section 5 , we use it to obtain some numerical results. Section 6 shows how the scheme in this paper can be used to approximate connecting orbits accurately and also contains an extension to strongly stable manifolds. Finally, we include an appendix which collects some useful results on Laguerre polynomials.

\section{VARIATIONAL, COLLOCATION AND EXPANSION FORMULATIONS}

Our aim in this section is to set up an efficient approximation scheme for (1.1) and (1.4). The key feature here is that this is a BVP over $[0, \infty)$ and such problems may be tackled in three ways.

- Truncate the interval to $[0, T]$ and use an approximate boundary condition at $T$.

- Transform the problem to a finite interval by a suitable mapping of the independent variable.

- Discretise the problem by using a set of approximating functions appropriate to $[0, \infty)$.

In 21] we looked at methods of the first two types; here we will consider type three. (The only similar paper we are aware of is [16, but the authors use a rational spectral method rather than our approach below.)

The natural space of approximating functions includes those of the form $e^{-\frac{\gamma}{2} t} \mathbf{q}(t)$, where $\gamma>0$ is a constant to be chosen and $\mathbf{q} \in X_{N}$ (the space of polynomials of degree $N$ or less with coefficients in $\mathbb{R}^{m}$ ). Thus the boundary condition at $t=\infty$ in (1.4) is automatically satisfied. In fact, it is convenient to consider $X_{N}$ approximating

where $\mathbf{u}^{\star}$ satisfies

$$
\mathbf{u}^{\star}(t) \equiv e^{\frac{\gamma}{2} t}\left\{\mathbf{v}^{\star}(t)-e^{\mathrm{A} t} \boldsymbol{\xi}\right\}
$$

$$
\mathbf{u}^{\prime}-\left[\frac{\gamma}{2} \mathbf{I}+\mathrm{A}\right] \mathbf{u}=e^{\frac{\gamma}{2} t} \mathbf{G}\left(e^{\mathrm{A} t} \boldsymbol{\xi}+e^{-\frac{\gamma}{2} t} \mathbf{u}\right)
$$

with side conditions

$$
P^{s} \mathbf{u}(0)=\mathbf{0} \quad \text { and } \quad \int_{0}^{\infty} e^{-\gamma t}\|\mathbf{u}(t)\|^{2} d t \quad \text { exists. }
$$

(I.e., the leading term of $\mathbf{v}^{\star}$ has been removed, but we are still constructing the stable manifold since $P^{u} \mathbf{u}^{\star}(0)=P^{u} \mathbf{v}^{\star}(0)$. In section 5, for comparison, we also exhibit results with this term left in.) We then introduce 
- our trial space $X_{N}^{0} \subset X_{N}$ to be that subspace satisfying the additional condition $P^{s} \mathbf{q}(0)=\mathbf{0}$,

- our test space $\bar{X}_{N} \subset X_{N}$ to be that subspace satisfying the additional condition $Q^{u} \mathbf{q} \in X_{N-1}$,

where $Q^{u}$ and $Q^{s}$ are the induced projections from the orthogonal analogue of (1.3), i.e.,

$$
\mathbb{R}^{m}=\mathcal{E}_{u}^{\perp} \oplus \mathcal{E}_{s}^{\perp} .
$$

Hence we would like to define our approximation $\mathbf{u}_{N}^{\star} \in X_{N}^{0}$ to satisfy the variational equations

$\int_{0}^{\infty} e^{-\gamma t}\left\{\mathbf{u}_{N}^{\prime}-\left[\frac{\gamma}{2} \mathbf{I}+\mathrm{A}\right] \mathbf{u}_{N}\right\} \circ \mathbf{q} d t=\int_{0}^{\infty} e^{-\gamma t}\left\{e^{\frac{\gamma}{2} t} \mathbf{G}\left(e^{\mathrm{A} t} \boldsymbol{\xi}+e^{-\frac{\gamma}{2} t} \mathbf{u}_{N}\right) \circ \mathbf{q}\right\} d t$

for every $\mathbf{q} \in \bar{X}_{N}$, where $\mathbf{x}_{1} \circ \mathbf{x}_{2}$ denotes the Euclidean inner-product on $\mathbb{R}^{m}$.

This may be simplified by the change of variable

$$
t \mapsto t / \gamma
$$

or equivalently the immediate change of independent variable in (1.1), replacing it with

$$
\mathbf{v}^{\prime}(t)=\frac{1}{\gamma} \mathbf{F}(\mathbf{v})
$$

(Note that this change of variable does not alter the value of the solution at $t=0$, which is the only part we are interested in.) Hence, remaining with the same notation $\mathbf{u}^{\star}$ and $\mathbf{u}_{N}^{\star}$ for convenience, we could define $\mathbf{u}_{N}^{\star} \in X_{N}^{0}$ approximating $\mathbf{u}^{\star}$ and satisfying

$$
\left\langle\mathbf{u}_{N}^{\prime}-\left[\frac{1}{2} \mathbf{I}+\frac{1}{\gamma} \mathrm{A}\right] \mathbf{u}_{N}, \mathbf{q}\right\rangle=\frac{1}{\gamma}\left\langle e^{t / 2} \mathbf{G}\left(e^{\frac{1}{\gamma} \mathrm{A} t} \boldsymbol{\xi}+e^{-t / 2} \mathbf{u}_{N}\right), \mathbf{q}\right\rangle
$$

for every $\mathbf{q} \in \bar{X}_{N}$, where

$$
\left\langle\mathbf{z}_{1}, \mathbf{z}_{2}\right\rangle \equiv \int_{0}^{\infty} e^{-t} \mathbf{z}_{1}(t) \circ \mathbf{z}_{2}(t) d t
$$

Thus, using the terminology of [5], we have a tau method for approximating the stable component of $\mathbf{u}^{\star}$ and a Galerkin method for the unstable component. This scheme, however, is impractical, because we cannot evaluate the r.h.s. of (2.5), and so instead we make use of

$$
\mathbf{x}_{1} \circ \mathbf{x}_{2}=P^{s} \mathbf{x}_{1} \circ Q^{u} \mathbf{x}_{2}+P^{u} \mathbf{x}_{1} \circ Q^{s} \mathbf{x}_{2}
$$

and we define $\mathbf{u}_{N}^{\star} \in X_{N}^{0}$ to be the solution of the approximate variational equations

$$
\left\langle\mathbf{u}_{N}^{\prime}-\left[\frac{1}{2} \mathbf{I}+\frac{1}{\gamma} \mathrm{A}\right] \mathbf{u}_{N}, \mathbf{q}\right\rangle=\frac{1}{\gamma}\left\langle e^{t / 2} \mathbf{G}\left(e^{\frac{1}{\gamma} \mathrm{A} t} \boldsymbol{\xi}+e^{-t / 2} \mathbf{u}_{N}\right), \mathbf{q}\right\rangle_{N}
$$

for every $\mathbf{q} \in \bar{X}_{N}$, where

$$
\left\langle\mathbf{z}_{1}, \mathbf{z}_{2}\right\rangle_{N} \equiv \sum_{i=1}^{N} w_{i} P^{s} \mathbf{z}_{1}\left(t_{i}\right) \circ Q^{u} \mathbf{z}_{2}\left(t_{i}\right)+\sum_{i=0}^{N} \widetilde{w}_{i} P^{u} \mathbf{z}_{1}\left(\tilde{t}_{i}\right) \circ Q^{s} \mathbf{z}_{2}\left(\tilde{t}_{i}\right) .
$$

These are the Gauss-Laguerre and Gauss-Laguerre-Radau quadrature points/ weights, which are defined in the Appendix, and we note that

$$
\left\langle\mathbf{q}_{1}, \mathbf{q}_{2}\right\rangle_{N}=\left\langle\mathbf{q}_{1}, \mathbf{q}_{2}\right\rangle \quad \forall \mathbf{q}_{1} \in X_{N}, \mathbf{q}_{2} \in \bar{X}_{N} .
$$


In section 3 we shall indeed prove that (2.7) has a locally unique solution. Finally, we emphasise again that $\gamma>0$ is a constant to be chosen by the user. We shall see, in section 5, how the accuracy of our stable manifold approximation depends on $\gamma$.

The above variational formulation is often useful theoretically. For actual computations, however, an equivalent collocation or modal formulation is more convenient.

2.1. Collocation framework. The collocation equations are obtained by inserting

$$
\mathbf{q}(t) \equiv \ell_{i}(t) \mathbf{x} \quad \forall \mathbf{x} \in \mathcal{E}_{u}^{\perp}, \quad i=1, \ldots, N
$$

where

$$
\ell_{i}(t) \equiv \frac{\prod_{\substack{j \neq i \\ j=1}}^{N}\left(t-t_{j}\right)}{\prod_{\substack{j \neq i \\ j=1}}^{N}\left(t_{i}-t_{j}\right)}, \quad i=1, \ldots, N
$$

are the Lagrange polynomials w.r.t. $\left\{t_{1}, \ldots, t_{N}\right\}$, and

$$
\mathbf{q}(t) \equiv \tilde{\ell}_{i}(t) \mathbf{x} \quad \forall \mathbf{x} \in \mathcal{E}_{s}^{\perp}, \quad i=0, \ldots, N,
$$

where

$$
\tilde{\ell}_{i}(t) \equiv \frac{\prod_{\substack{j \neq i \\ j=0}}^{N}\left(t-\tilde{t}_{j}\right)}{\prod_{\substack{j \neq i \\ j=0}}^{N}\left(\tilde{t}_{i}-\tilde{t}_{j}\right)}, \quad i=0, \ldots, N,
$$

are the Lagrange polynomials w.r.t. $\left\{\tilde{t}_{0}, \ldots, \tilde{t}_{N}\right\}$, in (2.7). Hence (2.7) is equivalent to

$$
\begin{aligned}
& P^{s} \mathbf{u}_{N}^{\prime}\left(t_{i}\right)-\left[\frac{1}{2} \mathrm{I}+\frac{1}{\gamma} \mathrm{A}\right] P^{s} \mathbf{u}_{N}\left(t_{i}\right) \\
& \quad=\frac{1}{\gamma} e^{t_{i} / 2} P^{s} \mathbf{G}\left(e^{\frac{1}{\gamma} \mathrm{A} t_{i}} \boldsymbol{\xi}+e^{-t_{i} / 2} \mathbf{u}_{N}\left(t_{i}\right)\right), \quad i=1, \ldots, N
\end{aligned}
$$

and

$$
\begin{aligned}
& P^{u} \mathbf{u}_{N}^{\prime}\left(\tilde{t}_{i}\right)-\left[\frac{1}{2} \mathbf{I}+\frac{1}{\gamma} \mathrm{A}\right] P^{u} \mathbf{u}_{N}\left(\tilde{t}_{i}\right) \\
& \quad=\frac{1}{\gamma} e^{\tilde{t}_{i} / 2} P^{u} \mathbf{G}\left(e^{\frac{1}{\gamma} \mathrm{A} \tilde{t}_{i}} \boldsymbol{\xi}+e^{-\tilde{t}_{i} / 2} \mathbf{u}_{N}\left(\tilde{t}_{i}\right)\right), \quad i=0, \ldots, N,
\end{aligned}
$$

with $P^{s} \mathbf{u}_{N}(0)=\mathbf{0}$. Defining the matrices

$$
\left(\mathrm{D}_{N}\right)_{i j}=d_{i j} \equiv\left[\frac{t}{t_{j}} \ell_{j}\right]^{\prime}\left(t_{i}\right), \quad i, j=1, \ldots, N,
$$

(i.e., zero is added to $\ell_{j}$ as an extra interpolation point) and

$$
\left(\widetilde{\mathrm{D}}_{N}\right)_{i j}=\tilde{d}_{i j} \equiv\left[\tilde{\ell}_{j}\right]^{\prime}\left(\tilde{t}_{i}\right), \quad i, j=0, \ldots, N
$$

so that

$$
P^{s} \mathbf{u}_{N}^{\prime}\left(t_{i}\right)=\sum_{j=1}^{N} d_{i j} P^{s} \mathbf{u}_{N}\left(t_{j}\right), \quad i=1, \ldots, N
$$

and

$$
P^{u} \mathbf{u}_{N}^{\prime}\left(\tilde{t}_{i}\right)=\sum_{j=0}^{N} \tilde{d}_{i j} P^{u} \mathbf{u}_{N}\left(\tilde{t}_{j}\right) . \quad i=0, \ldots, N
$$


our final discrete system satisfied by $\mathbf{u}_{N}^{\star}$ is

$$
\begin{aligned}
& \sum_{j=1}^{N} d_{i j} \mathbf{u}_{N}^{s}\left(t_{j}\right)-\left[\frac{1}{2} \mathbf{I}+\frac{1}{\gamma} \mathrm{A}\right] \mathbf{u}_{N}^{s}\left(t_{i}\right) \\
& =\frac{1}{\gamma} e^{t_{i} / 2} P^{s} \mathbf{G}\left(e^{\frac{1}{\gamma} A t_{i}} \boldsymbol{\xi}+e^{-t_{i} / 2} \mathbf{u}_{N}\left(t_{i}\right)\right), \\
& \sum_{j=0}^{N} \tilde{d}_{i j} \mathbf{u}_{N}^{u}\left(\tilde{t}_{j}\right)-\left[\frac{1}{2} \mathbf{I}+\frac{1}{\gamma} \mathrm{A}\right] \mathbf{u}_{N}^{u}\left(\tilde{t}_{i}\right) \\
& =\frac{1}{\gamma} e^{\tilde{t}_{i} / 2} P^{u} \mathbf{G}\left(e^{\frac{1}{\gamma} \mathrm{A} \tilde{t}_{i}} \boldsymbol{\xi}+e^{-\tilde{t}_{i} / 2} \mathbf{u}_{N}\left(\tilde{t}_{i}\right)\right), N,
\end{aligned}
$$

where $\mathbf{u}_{N}^{s} \equiv P^{s} \mathbf{u}_{N}$ and $\mathbf{u}_{N}^{u} \equiv P^{u} \mathbf{u}_{N}$. In section 4 we shall see how to efficiently solve this system of $(N+1) m$ equations and unknowns.

2.2. Laguerre expansion framework. Using the modal representation

$$
\mathbf{u}_{N}(t) \equiv \sum_{k=0}^{N} \mathbf{a}_{k} L_{k}(t)
$$

in (2.7), with $\mathbf{a}_{k}^{s} \equiv P^{s} \mathbf{a}_{k}$ and $\mathbf{a}_{k}^{u} \equiv P^{u} \mathbf{a}_{k}$, we have $\mathbf{u}_{N}^{s}(0)=\mathbf{0} \Longrightarrow \sum_{k=0}^{N} \mathbf{a}_{k}^{s}=\mathbf{0}$. Hence, inserting

$$
\mathbf{q}(t) \equiv \mathbf{x} L_{k}(t), \quad \mathbf{x} \in \mathcal{E}_{u}^{\perp}, \quad k=0, \ldots, N-1,
$$

and

$$
\mathbf{q}(t) \equiv \mathbf{x} L_{k}(t), \quad \mathbf{x} \in \mathcal{E}_{s}^{\perp}, \quad k=0, \ldots, N,
$$

in (2.7) and using (A.1b), (2.7) is equivalent to

$$
\begin{gathered}
\sum_{k=0}^{N} \mathbf{a}_{k}^{s}=\mathbf{0}, \\
\sum_{k=0}^{i-1} \mathbf{a}_{k}^{s}+\left[\frac{1}{2} \mathrm{l}-\frac{1}{\gamma} \mathrm{A}\right] \mathbf{a}_{i}^{s}=\frac{1}{\gamma} \mathbf{g}_{i}^{s}, \quad i=0, \ldots, N-1, \\
-\sum_{k=i+1}^{N} \mathbf{a}_{k}^{u}-\left[\frac{1}{2} \mathbf{l}+\frac{1}{\gamma} \mathrm{A}\right] \mathbf{a}_{i}^{u}=\frac{1}{\gamma} \mathbf{g}_{i}^{u}, \quad i=0, \ldots, N .
\end{gathered}
$$

Here

$$
\sum_{k=0}^{N-1} \mathbf{g}_{k}^{s} L_{k}(t)+\sum_{k=0}^{N} \mathbf{g}_{k}^{u} L_{k}(t) \equiv \widehat{I}_{N}\left\{e^{t / 2} \mathbf{G}\left(e^{\frac{1}{\gamma} A t} \boldsymbol{\xi}+e^{-t / 2} \mathbf{u}_{N}\right)\right\},
$$

where $\widehat{I}_{N}$ is the polynomial interpolation operator defined by:

- $\widehat{I}_{N}(\mathbf{z}) \equiv \widehat{I}_{N-1}^{G}\left(P^{s} \mathbf{z}\right)+I_{N}^{R}\left(P^{u} \mathbf{z}\right)$

- $\widehat{I}_{N-1}^{G}(\mathbf{z}) \in X_{N-1}$ with $\widehat{I}_{N-1}^{G}(\mathbf{z})\left(t_{i}\right)=\mathbf{z}\left(t_{i}\right), \quad i=1, \ldots, N$,

- $I_{N}^{R}(\mathbf{z}) \in X_{N}$ with $I_{N}^{R}(\mathbf{z})\left(\tilde{t}_{i}\right)=\mathbf{z}\left(\tilde{t}_{i}\right), \quad i=0, \ldots, N$. 
Again, in section 4 we shall see how to efficiently solve this system of $(N+1) m$ equations and unknowns.

2.3. Conclusion. We have chosen the quadrature rule (2.8) so that the three frameworks (2.7), (2.10) and (2.11) are all equivalent, in the sense of having the same solution $\mathbf{u}_{N}^{\star}$. (In fact, we shall see in section 4 that there are practical reasons for preferring the expansion formulation (2.11).) Of course, by breaking this equivalence, many slightly different discretisations are possible: e.g., we could just use Laguerre-Radau quadrature and replace (2.12) with full $N^{t h}$ degree interpolation at $\tilde{t}_{0}, \ldots, \tilde{t}_{N}$, which is not a collocation method. It would be easy to adapt the analysis in the next section to this scheme.

Finally, we note that, if the boundary condition at $t=0$ is left as inhomogeneous, i.e., (2.1) and (2.2) become

$$
\mathbf{u}^{\prime}-\left[\frac{\gamma}{2} \mathbf{I}+\mathrm{A}\right] \mathbf{u}=e^{\frac{\gamma}{2} t} \mathbf{G}\left(e^{-\frac{\gamma}{2} t} \mathbf{u}\right)
$$

with

$$
P^{s} \mathbf{u}(0)=\boldsymbol{\xi} \quad \text { and } \quad \int_{0}^{\infty} e^{-\gamma t} \mathbf{u}(t) \circ \mathbf{u}(t) d t \text { exists },
$$

then (2.11)-(2.12) are changed to

$$
\begin{gathered}
\sum_{k=0}^{N} \mathbf{a}_{k}^{s}=\boldsymbol{\xi}, \\
-\boldsymbol{\xi}+\sum_{k=0}^{i-1} \mathbf{a}_{k}^{s}+\left[\frac{1}{2} \mathbf{l}-\frac{1}{\gamma} \mathrm{A}\right] \mathbf{a}_{i}^{s}=\frac{1}{\gamma} \mathbf{g}_{i}^{s}, \quad i=0, \ldots, N-1, \\
-\sum_{k=i+1}^{N} \mathbf{a}_{k}^{u}-\left[\frac{1}{2} \mathbf{l}+\frac{1}{\gamma} \mathrm{A}\right] \mathbf{a}_{i}^{u}=\frac{1}{\gamma} \mathbf{g}_{i}^{u}, \quad i=0, \ldots, N,
\end{gathered}
$$

with

$$
\sum_{k=0}^{N-1} \mathbf{g}_{k}^{s} L_{k}(t)+\sum_{k=0}^{N} \mathbf{g}_{k}^{u} L_{k}(t) \equiv \widehat{I}_{N}\left\{e^{t / 2} \mathbf{G}\left(e^{-t / 2} \mathbf{u}_{N}\right)\right\} .
$$

In section 5 we shall compare the numerical results for (2.13)-(2.14) with those for (2.11)-(2.12).

\section{EXISTENCE, UNIQUENESS AND ERROR OF APPROXIMATION}

We first introduce an appropriate norm on $\mathbb{R}^{m}$, with respect to which the matrix A has suitable definiteness properties. To do this, we require the following standard lemma, where

$$
0<\lambda^{s} \equiv \min \{-\Re \mathrm{e}(\lambda): \quad \lambda \text { an eigenvalue of A with } \Re \mathrm{e}(\lambda)<0\}
$$

and

$$
0<\lambda^{u} \equiv \min \{\Re \mathrm{e}(\lambda): \quad \lambda \text { an eigenvalue of } \mathrm{A} \text { with } \Re \mathrm{e}(\lambda)>0\}
$$

measure the hyperbolicity of the origin. 
Lemma. For any $\varepsilon>0$ there exists a symmetric positive-definite $\mathrm{M}_{\varepsilon} \in \mathbb{R}^{m \times m}$, with

such that

$$
\mathrm{M}_{\varepsilon}: \mathcal{E}_{s} \mapsto \mathcal{E}_{u}^{\perp} \quad \text { and } \quad \mathrm{M}_{\varepsilon}: \mathcal{E}_{u} \mapsto \mathcal{E}_{s}^{\perp}
$$

$$
\begin{array}{rlrl}
-\mathbf{x} \circ \mathrm{M}_{\varepsilon} A \mathbf{x} & \geq\left(\lambda^{s}-\varepsilon\right) \mathbf{x} \circ \mathrm{M}_{\varepsilon} \mathbf{x} & & \forall \mathbf{x} \in \mathcal{E}_{s}, \\
\mathbf{x} \circ \mathrm{M}_{\varepsilon} A \mathbf{x} \geq\left(\lambda^{u}-\varepsilon\right) \mathbf{x} \circ \mathrm{M}_{\varepsilon} \mathbf{x} & & \forall \mathbf{x} \in \mathcal{E}_{u} .
\end{array}
$$

Proof. See [15. We note that $\mathrm{M}_{\varepsilon}$ may be constructed using a basis of eigenvectors, if this exists. The condition of $M_{\varepsilon}$, however, will blow up as $\varepsilon \rightarrow 0$ if a critical eigenvalue of $A$ is defective; otherwise $\varepsilon=0$ is also possible.

Hence we are able to assert the following theorem, where the $\mathrm{A}_{s / u}$ denote the restrictions of $\mathrm{A}$ to $\mathcal{E}_{s}$ and $\mathcal{E}_{u}$.

Theorem. There exists a symmetric positive-definite $\mathrm{M} \in \mathbb{R}^{m \times m}$, hence defining a norm

$$
\|\mathrm{x}\|_{\mathrm{M}} \equiv \sqrt{\mathrm{x} \circ \mathrm{Mx}}
$$

which satisfies $\mathrm{M}: \mathcal{E}_{s} \mapsto \mathcal{E}_{u}^{\perp}$ and $\mathrm{M}: \mathcal{E}_{u} \mapsto \mathcal{E}_{s}^{\perp}$, and also the following properties:

- $\exists c^{s}>0$ such that

$$
-\mathbf{x} \circ \mathrm{MAx} \geq c^{s}\|\mathbf{x}\|_{\mathrm{M}}^{2} \quad \forall \mathbf{x} \in \mathcal{E}_{s},
$$

- $\exists c^{u}>0$ such that

$$
\mathbf{x} \circ \mathrm{MAx} \geq c^{u}\|\mathbf{x}\|_{\mathrm{M}}^{2} \quad \forall \mathbf{x} \in \mathcal{E}_{u},
$$

- for each $\gamma>0, \exists \theta_{\gamma}^{s}<1$ such that

$$
\left\|\left[\frac{\gamma}{2} \mathbf{l}-\mathrm{A}_{s}\right]^{-1}\left[\frac{\gamma}{2} \mathbf{I}+\mathrm{A}_{s}\right]\right\|_{\mathrm{M}} \leq \theta_{\gamma}^{s},
$$

- for each $\gamma>0, \exists \theta_{\gamma}^{u}<1$ such that

$$
\left\|\left[\frac{\gamma}{2} \mathbf{I}+\mathrm{A}_{u}\right]^{-1}\left[\frac{\gamma}{2} \mathbf{I}-\mathrm{A}_{u}\right]\right\|_{\mathrm{M}} \leq \theta_{\gamma}^{u}
$$

Proof. The first two parts are simply the above lemma. The last two parts follow from

$$
\rho\left(\left[\frac{\gamma}{2} \mathbf{I}-\mathrm{A}_{s}\right]^{-1}\left[\frac{\gamma}{2} \mathbf{I}+\mathrm{A}_{s}\right]\right)<1 \text { and } \rho\left(\left[\frac{\gamma}{2} \mathbf{I}+\mathrm{A}_{u}\right]^{-1}\left[\frac{\gamma}{2} \mathbf{I}-\mathrm{A}_{u}\right]\right)<1
$$

and the fact that the construction of $M_{\varepsilon}$ in the proof of the above lemma also provides a norm approximating the spectral radius.

We note that $\theta_{\gamma}^{s / u} \rightarrow 1$ as $\gamma \rightarrow 0$ or $\gamma \rightarrow \infty$.

3.1. Bounds for the linear problem. In this subsection, we shall bound the solution of the linear analogue of (2.7), i.e.,

$$
\left\langle\mathbf{u}_{N}^{\prime}-\left[\frac{1}{2} \mathbf{I}+\frac{1}{\gamma} \mathrm{A}\right] \mathbf{u}_{N}, \mathbf{q}\right\rangle=\frac{1}{\gamma}\left\langle e^{t / 2} \mathbf{f}(t), \mathbf{q}\right\rangle_{N}
$$

for every $\mathbf{q} \in \bar{X}_{N}$, by explicitly using Laguerre expansions. Thus, setting

$$
\begin{aligned}
& \mathbf{u}_{N} \equiv \sum_{k=0}^{N} \mathbf{a}_{k} L_{k}(t), \quad \mathbf{u}_{N}^{\prime} \equiv \sum_{k=0}^{N-1} \mathbf{b}_{k} L_{k}(t), \\
& \widehat{I}_{N}\left(e^{t / 2} \mathbf{f}(t)\right) \equiv \sum_{k=0}^{N-1} \mathbf{f}_{k}^{s} L_{k}(t)+\sum_{k=0}^{N} \mathbf{f}_{k}^{u} L_{k}(t),
\end{aligned}
$$


where $\mathbf{f}^{s} \equiv P^{s} \mathbf{f}, \mathbf{f}^{u} \equiv P^{u} \mathbf{f}$ and $\mathbf{f}_{k} \equiv \mathbf{f}_{k}^{s}+\mathbf{f}_{k}^{u}$, (3.1) becomes

$$
\begin{aligned}
\mathbf{b}_{k}-\left[\frac{1}{2} \mathbf{I}+\frac{1}{\gamma} \mathrm{A}\right] \mathbf{a}_{k} & =\frac{1}{\gamma} \mathbf{f}_{k}, \quad k=0, \ldots, N-1, \\
-\left[\frac{1}{2} \mathbf{I}+\frac{1}{\gamma} \mathrm{A}_{u}\right] \mathbf{a}_{N}^{u} & =\frac{1}{\gamma} \mathbf{f}_{N}^{u}, \\
\sum_{k=0}^{N} \mathbf{a}_{k}^{s} & =\mathbf{0} .
\end{aligned}
$$

Now we will consider the stable and unstable components of (3.2) separately.

3.1.1. Stable components. Using (A.1b) in (3.2) gives the lower triangular system

$$
\begin{gathered}
{\left[\frac{1}{2} \mathbf{l}-\frac{1}{\gamma} \mathrm{A}_{s}\right] \mathbf{a}_{k}^{s}+\sum_{j=0}^{k-1} \mathbf{a}_{j}^{s}=\frac{1}{\gamma} \mathbf{f}_{k}^{s}, \quad k=0, \ldots, N-1,} \\
\sum_{k=0}^{N} \mathbf{a}_{k}^{s}=\mathbf{0},
\end{gathered}
$$

which is invertible since $\left[\frac{1}{2} \mathrm{I}-\frac{1}{\gamma} \mathrm{A}_{s}\right]$ is nonsingular. Hence, if we multiply (3.3a) by $\mathrm{Ma}_{k}^{s}$, sum over $k=0, \ldots, N-1$ and use the fact that

$$
\sum_{k=0}^{N-1}\left(\mathbf{M a}_{k}^{s}\right) \circ\left(\sum_{j=0}^{k-1} \mathbf{a}_{j}^{s}\right)=\frac{1}{2}\left\|\mathbf{a}_{N}^{s}\right\|_{\mathrm{M}}^{2}-\frac{1}{2} \sum_{k=0}^{N-1}\left\|\mathbf{a}_{k}^{s}\right\|_{\mathrm{M}}^{2},
$$

we obtain

$$
\frac{\gamma}{2}\left\|\mathbf{a}_{N}^{s}\right\|_{\mathrm{M}}^{2}-\sum_{k=0}^{N-1}\left(\mathrm{Ma}_{k}^{s}\right) \circ\left(\mathrm{A}_{s} \mathbf{a}_{k}^{s}\right)=\sum_{k=0}^{N-1}\left(\mathbf{M a}_{k}^{s}\right) \circ \mathbf{f}_{k}^{s} .
$$

Finally, applying Cauchy-Schwarz to the r.h.s. of (3.4) and utilising the above theorem and (3.3b), we arrive at the bound

$$
\left(\sum_{k=0}^{N}\left\|\mathbf{a}_{k}^{s}\right\|_{\mathrm{M}}^{2}\right)^{1 / 2} \leq \frac{1}{\min \left\{\gamma / 2, c^{s}\right\}}\left(\sum_{k=0}^{N-1}\left\|\mathbf{f}_{k}^{s}\right\|_{\mathrm{M}}^{2}\right)^{1 / 2} .
$$

We may also apply (A.1a) to (3.2) to obtain the lower bidiagonal system

$$
\begin{aligned}
& {\left[\frac{1}{2} \mathbf{I}-\frac{1}{\gamma} \mathrm{A}_{s}\right] \mathbf{b}_{0}^{s} }=\frac{1}{\gamma} \mathbf{f}_{0}^{s}, \\
& {\left[\frac{1}{2} \mathbf{l}-\frac{1}{\gamma} \mathrm{A}_{s}\right] \mathbf{b}_{k}^{s}-\left[\frac{1}{2} \mathbf{I}+\frac{1}{\gamma} \mathrm{A}_{s}\right] \mathbf{b}_{k-1}^{s}=\frac{1}{\gamma} \mathbf{f}_{k}^{s}, \quad k=1, \ldots, N-1 . }
\end{aligned}
$$

Hence

$$
\begin{aligned}
\mathbf{b}_{0}^{s} & =\left[\frac{\gamma}{2} \mathbf{l}-\mathrm{A}_{s}\right]^{-1} \mathbf{f}_{0}^{s}, \\
\mathbf{b}_{k}^{s}-\left[\frac{\gamma}{2} \mathbf{l}-\mathrm{A}_{s}\right]^{-1}\left[\frac{\gamma}{2} \mathbf{l}+\mathrm{A}_{s}\right] \mathbf{b}_{k-1}^{s} & =\left[\frac{\gamma}{2} \mathbf{l}-\mathrm{A}_{s}\right]^{-1} \mathbf{f}_{k}^{s}, \quad k=1, \ldots, N-1,
\end{aligned}
$$

and, since $\theta_{\gamma}^{s}<1$ in the above theorem, a standard perturbation-of-the-identity argument gives us the bound

$$
\left(\sum_{k=0}^{N-1}\left\|\mathbf{b}_{k}^{s}\right\|_{\mathrm{M}}^{2}\right)^{1 / 2} \leq \frac{\left\|\left[\frac{\gamma}{2} \mathbf{l}-\mathrm{A}_{s}\right]^{-1}\right\|_{\mathrm{M}}}{1-\theta_{\gamma}^{s}}\left(\sum_{k=0}^{N-1}\left\|\mathbf{f}_{k}^{s}\right\|_{\mathrm{M}}^{2}\right)^{1 / 2}
$$


3.1.2. Unstable components. Using (A.1b) in (3.2) gives the upper triangular system

$$
-\left[\frac{1}{2} \mathrm{l}+\frac{1}{\gamma} \mathrm{A}_{u}\right] \mathbf{a}_{k}^{u}-\sum_{j=k+1}^{N} \mathbf{a}_{j}^{u}=\frac{1}{\gamma} \mathbf{f}_{k}^{u}, \quad k=N, \ldots, 0,
$$

which is invertible since $\left[\frac{1}{2} \mathrm{I}+\frac{1}{\gamma} \mathrm{A}_{u}\right]$ is nonsingular. Hence, multiplying (3.8) by $\mathbf{M a}_{k}^{u}$, summing over $k=0, \ldots, N$, and using the fact that

$$
\begin{aligned}
\sum_{k=0}^{N}\left(\mathrm{M} \mathbf{a}_{k}^{u}\right) \circ\left(\sum_{j=k+1}^{N} \mathbf{a}_{j}^{u}\right) & =\frac{1}{2}\left\|\sum_{k=0}^{N} \mathbf{a}_{k}^{u}\right\|_{\mathrm{M}}^{2}-\frac{1}{2} \sum_{k=0}^{N}\left\|\mathbf{a}_{k}^{u}\right\|_{\mathrm{M}}^{2} \\
& =\frac{1}{2}\left\|P^{u} \mathbf{u}_{N}(0)\right\|_{\mathrm{M}}^{2}-\frac{1}{2} \sum_{k=0}^{N}\left\|\mathbf{a}_{k}^{u}\right\|_{\mathrm{M}}^{2},
\end{aligned}
$$

we obtain

$$
-\frac{\gamma}{2}\left\|P^{u} \mathbf{u}_{N}(0)\right\|_{\mathrm{M}}^{2}-\sum_{k=0}^{N}\left(\mathrm{Ma}_{k}^{u}\right) \circ\left(\mathrm{A}_{u} \mathbf{a}_{k}^{u}\right)=\sum_{k=0}^{N}\left(\mathrm{Ma}_{k}^{u}\right) \circ \mathbf{f}_{k}^{u} .
$$

Finally, applying Cauchy-Schwarz to the r.h.s. of (3.9) and utilising the above theorem, we arrive at the bound

$$
\left(\sum_{k=0}^{N}\left\|\mathbf{a}_{k}^{u}\right\|_{\mathrm{M}}^{2}\right)^{1 / 2} \leq \frac{1}{c^{u}}\left(\sum_{k=0}^{N}\left\|\mathbf{f}_{k}^{u}\right\|_{\mathrm{M}}^{2}\right)^{1 / 2} .
$$

We may also apply A.1a to (3.2) to obtain the upper bidiagonal system

$$
\begin{aligned}
& {\left[\frac{1}{2} \mathbf{l}+\frac{1}{\gamma} \mathrm{A}_{u}\right] \mathbf{b}_{N-1}^{u} }=\frac{1}{\gamma} \mathbf{f}_{N}^{u}, \\
& {\left[\frac{1}{2} \mathbf{l}+\frac{1}{\gamma} \mathrm{A}_{u}\right] \mathbf{b}_{k}^{u}+\left[\frac{1}{2} \mathbf{l}-\frac{1}{\gamma} \mathrm{A}_{u}\right] \mathbf{b}_{k-1}^{u}=\frac{1}{\gamma} \mathbf{f}_{k}^{u}, \quad k=N-1, \ldots, 1 . }
\end{aligned}
$$

Hence

$$
\begin{aligned}
\mathbf{b}_{N-1}^{u} & =\left[\frac{\gamma}{2} \mathbf{I}+\mathrm{A}_{u}\right]^{-1} \mathbf{f}_{N}^{u}, \\
\mathbf{b}_{k-1}^{u}-\left[\frac{\gamma}{2} \mathbf{I}+\mathrm{A}_{u}\right]^{-1}\left[\frac{\gamma}{2} \mathbf{I}-\mathrm{A}_{u}\right] \mathbf{b}_{k}^{u}, & =\left[\frac{\gamma}{2} \mathbf{I}+\mathrm{A}_{u}\right]^{-1} \mathbf{f}_{k}^{u}, \quad k=N-1, \ldots, 1,
\end{aligned}
$$

and so $\theta_{\gamma}^{u}<1$ in the above theorem gives us the bound

$$
\left(\sum_{k=0}^{N-1}\left\|\mathbf{b}_{k}^{u}\right\|_{\mathrm{M}}^{2}\right)^{1 / 2} \leq \frac{\left\|\left[\frac{\gamma}{2} \mathbf{l}+\mathrm{A}_{u}\right]^{-1}\right\|_{\mathrm{M}}}{1-\theta_{\gamma}^{u}}\left(\sum_{k=1}^{N}\left\|\mathbf{f}_{k}^{u}\right\|_{\mathrm{M}}^{2}\right)^{1 / 2} .
$$

3.1.3. Conclusion. We now bound the solution of (3.1) by amalgamating the results of subsections 3.1.1 and 3.1.2. If we switch to the 2-norm, combining (3.5) and (3.7) gives

$$
\left(\sum_{k=0}^{N}\left\|\mathbf{a}_{k}^{s}\right\|^{2}+\sum_{k=0}^{N-1}\left\|\mathbf{b}_{k}^{s}\right\|^{2}\right)^{1 / 2} \leq C_{\gamma}^{s}\left(\sum_{k=0}^{N-1}\left\|\mathbf{f}_{k}^{s}\right\|^{2}\right)^{1 / 2},
$$

where

$$
C_{\gamma}^{s} \equiv \sqrt{\|\mathrm{M}\|\left\|\mathrm{M}^{-1}\right\|}\left\{\frac{1}{\min \left\{\gamma / 2, c^{s}\right\}}+\frac{\left\|\left[\frac{\gamma}{2} \mathrm{I}-\mathrm{A}_{s}\right]^{-1}\right\|_{\mathrm{M}}}{1-\theta_{\gamma}^{s}}\right\}
$$


while combining (3.10) and (3.12) gives

$$
\left(\sum_{k=0}^{N}\left\|\mathbf{a}_{k}^{u}\right\|^{2}+\sum_{k=0}^{N-1}\left\|\mathbf{b}_{k}^{u}\right\|^{2}\right)^{1 / 2} \leq C_{\gamma}^{u}\left(\sum_{k=0}^{N}\left\|\mathbf{f}_{k}^{u}\right\|^{2}\right)^{1 / 2}
$$

where

$$
C_{\gamma}^{u} \equiv \sqrt{\|\mathrm{M}\|\left\|\mathrm{M}^{-1}\right\|}\left\{\frac{1}{c^{u}}+\frac{\left\|\left[\frac{\gamma}{2} \mathbf{I}+\mathrm{A}_{u}\right]^{-1}\right\|_{\mathrm{M}}}{1-\theta_{\gamma}^{u}}\right\} .
$$

Hence, since the equivalences

$$
\begin{array}{rlrl}
\int_{0}^{\infty} e^{-t}\left\|\mathbf{u}_{N}(t)\right\|^{2} d t & \equiv \sum_{k=0}^{N}\left\|\mathbf{a}_{k}\right\|^{2}, & & \sum_{i=1}^{N} w_{i} e^{t_{i}}\left\|\mathbf{f}^{s}\left(t_{i}\right)\right\|^{2} \equiv \sum_{k=0}^{N-1}\left\|\mathbf{f}_{k}^{s}\right\|^{2}, \\
\int_{0}^{\infty} e^{-t}\left\|\mathbf{u}_{N}^{\prime}(t)\right\|^{2} d t \equiv \sum_{k=0}^{N-1}\left\|\mathbf{b}_{k}\right\|^{2}, & & \sum_{i=0}^{N} \widetilde{w}_{i} e^{\tilde{e}_{i}}\left\|\mathbf{f}^{u}\left(\tilde{t}_{i}\right)\right\|^{2} \equiv \sum_{k=0}^{N}\left\|\mathbf{f}_{k}^{u}\right\|^{2}
\end{array}
$$

are just vector forms of (A.1c), (A.2c), and (A.2d), we may use (A.5) to show $\exists C_{\gamma}>0$ such that

$$
\left\|\left|\mathbf{u}_{N} \|\right|_{H^{1}} \leq \frac{C_{\gamma}}{1-\beta} \max _{t \geq 0}\left\{e^{-\beta t / 2} e^{t / 2}\|\mathbf{f}(t)\|\right\}\right.
$$

for each $\beta \in[0,1)$, where

$$
\left.\|\mathbf{z}\|\right|_{H^{1}} \equiv\left(\int_{0}^{\infty} e^{-t}\left\{\|\mathbf{z}(t)\|^{2}+\left\|\mathbf{z}^{\prime}(t)\right\|^{2}\right\} d t\right)^{1 / 2} .
$$

We shall also need the following Sobolev inequality from [17:

- $\exists C_{S} \geq 1$ such that

$$
\left.\left\|\left|\mathbf{z}_{N}\left\|\left.\right|_{L^{\infty}} \leq C_{S}\right\|\right| \mathbf{z}_{N}\right\|\right|_{H^{1}}
$$

holds for all $\mathbf{z} \in X_{N}$ and all $N>0$, where

$$
\left\||\mathbf{z} \||_{L^{\infty}} \equiv \max _{t \geq 0}\left\{e^{-t / 2}\|\mathbf{z}(t)\|\right\} .\right.
$$

Thus, from (3.15), we also have the bound

$$
\left.\left\|\mathbf{u}_{N}\right\|\right|_{L^{\infty}} \leq \frac{C_{S} C_{\gamma}}{1-\beta} \max _{t \geq 0}\left\{e^{-\beta t / 2} e^{t / 2}\|\mathbf{f}(t)\|\right\}
$$

Hence we have shown existence and uniqueness for the solution of the discrete linear problem (3.1) and obtained the bounds (3.15) and (3.16).

3.2. Solution of the nonlinear problem. Now we shall use the bounds of section 3.1 to prove the existence and local uniqueness of a solution for the nonlinear equation (2.7), i.e.,

$$
\left\langle\mathbf{u}_{N}^{\prime}-\left[\frac{1}{2} \mathbf{I}+\frac{1}{\gamma} \mathrm{A}\right] \mathbf{u}_{N}, \mathbf{q}\right\rangle=\frac{1}{\gamma}\left\langle e^{t / 2} \mathbf{G}\left(e^{\frac{1}{\gamma} \mathrm{A} t} \boldsymbol{\xi}+e^{-t / 2} \mathbf{u}_{N}\right), \mathbf{q}\right\rangle_{N}
$$

for every $\mathbf{q} \in \bar{X}_{N}$. To achieve this, we must be more specific about the exponential decay of $e^{\mathrm{A}_{s} t}$ and make an assumption about the behaviour of the higher-order term $\mathbf{G}$ in (1.2). 
- $\exists \alpha_{\gamma} \in\left(0, \frac{1}{2}\right]$ and $\exists C_{\mathrm{A}} \geq 1$ such that

$$
\left\|e^{\frac{1}{\gamma} \mathrm{A} t} P^{s}\right\| \leq C_{\mathrm{A}} e^{-\alpha_{\gamma} t} \quad \forall t \geq 0 .
$$

Note that, if a critical eigenvalue of $\mathrm{A}_{s}$ is not defective, we may choose $\alpha_{\gamma} \equiv \min \left\{\lambda^{s} / \gamma, 1 / 2\right\}$.

- $K(r)$ is a nondecreasing function of $r$ such that, $\forall\left\|\mathbf{x}_{1}\right\|,\left\|\mathbf{x}_{2}\right\| \leq r$,

$$
\left\|\mathbf{G}\left(\mathbf{x}_{1}\right)-\mathbf{G}\left(\mathbf{x}_{2}\right)\right\| \leq K(r) \max \left\{\left\|\mathbf{x}_{1}\right\|,\left\|\mathbf{x}_{2}\right\|\right\}\left\|\mathbf{x}_{1}-\mathbf{x}_{2}\right\| .
$$

Now we define $r^{\star}$ by

$$
\frac{C_{S} C_{\gamma}}{2 \alpha_{\gamma}} K\left(2 C_{\mathrm{A}} r^{\star}\right) 4 C_{\mathrm{A}} r^{\star}=1,
$$

and consider the mapping $\mathbf{u}_{N} \mapsto \mathbf{z}_{N} \equiv \mathcal{K}\left(\mathbf{u}_{N}\right) \in X_{N}^{0}$ defined by

$$
\left\langle\mathbf{z}_{N}^{\prime}-\left[\frac{1}{2} \mathbf{I}+\frac{1}{\gamma} \mathrm{A}\right] \mathbf{z}_{N}, \mathbf{q}\right\rangle=\frac{1}{\gamma}\left\langle e^{t / 2} \mathbf{G}\left(e^{\frac{1}{\gamma} A t} \boldsymbol{\xi}+e^{-t / 2} \mathbf{u}_{N}\right), \mathbf{q}\right\rangle_{N}
$$

for every $\mathbf{q} \in \bar{X}_{N}$.

Theorem. If $\|\boldsymbol{\xi}\| \leq r^{\star}$, then $\mathcal{K}$ is a contraction on the set

$$
\mathcal{S} \equiv\left\{\mathbf{u}_{N} \in X_{N}^{0}:\left\|\mathbf{u}_{N} \mid\right\|_{L^{\infty}} \leq C_{\mathrm{A}}\|\boldsymbol{\xi}\|\right\}
$$

with respect to $\||\cdot|\|_{L^{\infty}}$.

Proof. We set $\beta \equiv 1-2 \alpha_{\gamma}$ in (3.16). Hence $\mathcal{K}\left(\mathbf{u}_{N}\right) \in \mathcal{S}$ since

$$
\begin{aligned}
\left\|\left|\mathcal{K}\left(\mathbf{u}_{N}\right)\right|\right\|_{L^{\infty}} & \leq \frac{C_{S} C_{\gamma}}{2 \alpha_{\gamma}} \max _{t \geq 0}\left\{e^{\alpha_{\gamma} t}\left\|\mathbf{G}\left(e^{\frac{1}{\gamma} \mathrm{A} t} \boldsymbol{\xi}+e^{-t / 2} \mathbf{u}_{N}(t)\right)\right\|\right\} \\
& \leq \frac{C_{S} C_{\gamma}}{2 \alpha_{\gamma}} K\left(2 C_{\mathrm{A}}\|\boldsymbol{\xi}\|\right)\left[2 C_{\mathrm{A}}\|\boldsymbol{\xi}\|\right]^{2} \\
& \leq C_{\mathrm{A}}\|\boldsymbol{\xi}\| .
\end{aligned}
$$

Also $\mathcal{K}$ is a contraction on $\mathcal{S}$ since

$$
\begin{aligned}
\left\|\left|\mathcal{K}\left(\mathbf{u}_{N}\right)-\mathcal{K}\left(\mathbf{v}_{N}\right)\right|\right\|_{L^{\infty}} \\
\quad \leq \frac{C_{S} C_{\gamma}}{2 \alpha_{\gamma}} \max _{t \geq 0}\left\{e^{\alpha_{\gamma} t}\left\|\mathbf{G}\left(e^{\frac{1}{\gamma} A t} \boldsymbol{\xi}+e^{-t / 2} \mathbf{u}_{N}(t)\right)-\mathbf{G}\left(e^{\frac{1}{\gamma} A t} \boldsymbol{\xi}+e^{-t / 2} \mathbf{v}_{N}(t)\right)\right\|\right\} \\
\quad \leq\left\{\frac{C_{S} C_{\gamma}}{2 \alpha_{\gamma}} K\left(2 C_{\mathrm{A}}\|\boldsymbol{\xi}\|\right) 2 C_{\mathrm{A}}\|\boldsymbol{\xi}\|\right\}\left\|\left|\mathbf{u}_{N}-\mathbf{v}_{N}\right|\right\|_{L^{\infty}} \\
\quad \leq \frac{1}{2}\left\|\left|\mathbf{u}_{N}-\mathbf{v}_{N} \|\right|_{L^{\infty}} .\right.
\end{aligned}
$$

Thus the Contraction Mapping Theorem guarantees a locally unique solution of (3.17), which we denote by $\mathbf{u}_{N}^{\star}$.

3.3. Basic error bound. Let $I_{N}$ be the polynomial interpolation operator defined by

where

$$
I_{N}(\mathbf{z}) \equiv I_{N}^{G}\left(P^{s} \mathbf{z}\right)+I_{N}^{R}\left(P^{u} \mathbf{z}\right),
$$

$$
I_{N}^{G}(\mathbf{z}) \in X_{N} \quad \text { with } I_{N}^{G}(\mathbf{z})\left(t_{i}\right)=\mathbf{z}\left(t_{i}\right) \quad i=0, \ldots, N,
$$

with $t_{0} \equiv 0$; and denote $\mathbf{u}_{I}^{\star} \equiv I_{N}\left(\mathbf{u}^{\star}\right)$. Then $\mathbf{u}_{I}^{\star}$ satisfies

$$
\left\langle I_{N}\left(\mathbf{u}^{\star^{\prime}}\right)-\left[\frac{1}{2} \mathbf{l}+\frac{1}{\gamma} \mathrm{A}\right] \mathbf{u}_{I}^{\star}, \mathbf{q}\right\rangle=\frac{1}{\gamma}\left\langle e^{t / 2} \mathbf{G}\left(e^{\frac{1}{\gamma} \mathrm{A} t} \boldsymbol{\xi}+e^{-t / 2} \mathbf{u}^{\star}\right), \mathbf{q}\right\rangle_{N}
$$


for every $\mathbf{q} \in \bar{X}_{N}$, since

$$
\left\langle I_{N}\left(\mathbf{u}^{\star^{\prime}}\right)-\left[\frac{1}{2} \mathbf{l}+\frac{1}{\gamma} \mathrm{A}\right] \mathbf{u}_{I}^{\star}, \mathbf{q}\right\rangle=\left\langle\mathbf{u}^{\star^{\prime}}-\left[\frac{1}{2} \mathbf{l}+\frac{1}{\gamma} \mathrm{A}\right] \mathbf{u}^{\star}, \mathbf{q}\right\rangle_{N}
$$

for all $\mathbf{q} \in \bar{X}_{N}$. Hence subtracting (3.19) from

$$
\left\langle\mathbf{u}_{N}^{\star}{ }^{\prime}-\left[\frac{1}{2} \mathbf{I}+\frac{1}{\gamma} \mathrm{A}\right] \mathbf{u}_{N}^{\star}, \mathbf{q}\right\rangle=\frac{1}{\gamma}\left\langle e^{t / 2} \mathbf{G}\left(e^{\frac{1}{\gamma} \mathrm{A} t} \boldsymbol{\xi}+e^{-t / 2} \mathbf{u}_{N}^{\star}\right), \mathbf{q}\right\rangle_{N}
$$

gives

$$
\begin{aligned}
& \left\langle\left(\mathbf{u}_{N}^{\star}-\mathbf{u}_{I}^{\star}\right)^{\prime}-\left[\frac{1}{2} \mathbf{I}+\frac{1}{\gamma} \mathrm{A}\right]\left(\mathbf{u}_{N}^{\star}-\mathbf{u}_{I}^{\star}\right), \mathbf{q}\right\rangle \\
& =\frac{1}{\gamma}\left\langle e^{t / 2}\left\{\mathbf{G}\left(e^{\frac{1}{\gamma} \mathrm{A} t} \boldsymbol{\xi}+e^{-t / 2} \mathbf{u}_{N}^{\star}\right)-\mathbf{G}\left(e^{\frac{1}{\gamma} \mathrm{A} t} \boldsymbol{\xi}+e^{-t / 2} \mathbf{u}^{\star}\right)\right\}, \mathbf{q}\right\rangle_{N} \\
& +\left\langle I_{N}\left(\mathbf{u}^{\star^{\prime}}\right)-\mathbf{u}_{I}^{\star^{\prime}}, \mathbf{q}\right\rangle
\end{aligned}
$$

for every $\mathbf{q} \in \bar{X}_{N}$. Now, if we assume that

$$
\left\|\left|\mathbf{u}^{\star}\right|\right\|_{L^{\infty}} \leq C_{\mathrm{A}}\|\boldsymbol{\xi}\|
$$

which is certainly true for $\|\boldsymbol{\xi}\|$ sufficiently small, and combine the arguments in the proof of the above theorem with those leading to (3.15), we obtain

$$
\begin{aligned}
\left\|\left|\mathbf{u}_{N}^{\star}-\mathbf{u}_{I}^{\star}\right|\right\|_{H^{1}} \leq\left\{\frac{C_{\gamma}}{2 \alpha_{\gamma}} K\left(2 C_{\mathrm{A}}\|\boldsymbol{\xi}\|\right)\right. & \left.2 C_{\mathrm{A}}\|\boldsymbol{\xi}\|\right\}\left\|\left|\mathbf{u}_{N}^{\star}-\mathbf{u}^{\star}\right|\right\|_{L^{\infty}} \\
& +\frac{\gamma C_{\gamma}}{1-\beta} \max _{t \geq 0}\left\{e^{-\beta t / 2}\left\|\mathbf{u}^{\star^{\prime}}(t)-\mathbf{u}_{I}^{\star \prime}(t)\right\|\right\}
\end{aligned}
$$

and, from the Sobolev inequality,

$$
\begin{aligned}
\left\|\left|\mathbf{u}_{N}^{\star}-\mathbf{u}_{I}^{\star}\right|\right\|_{L^{\infty}} \leq\left\{\frac{C_{S} C_{\gamma}}{2 \alpha_{\gamma}} K\left(2 C_{\mathrm{A}}\|\boldsymbol{\xi}\|\right) 2 C_{\mathrm{A}}\|\boldsymbol{\xi}\|\right\}\left\|\left|\mathbf{u}_{N}^{\star}-\mathbf{u}^{\star}\right|\right\|_{L^{\infty}} \\
+\frac{\gamma C_{S} C_{\gamma}}{1-\beta} \max _{t \geq 0}\left\{e^{-\beta t / 2}\left\|\mathbf{u}^{\star^{\prime}}(t)-\mathbf{u}_{I}^{\star^{\prime}}(t)\right\|\right\}
\end{aligned}
$$

for each $\beta \in[0,1)$. Consequently, writing

$$
\begin{aligned}
\left\|\left|\mathbf{u}_{N}^{\star}-\mathbf{u}^{\star}\right|\right\|_{L^{\infty}} & \leq\left\|\left|\mathbf{u}_{N}^{\star}-\mathbf{u}_{I}^{\star}\right|\right\|_{L^{\infty}}+\left\|\left|\mathbf{u}_{I}^{\star}-\mathbf{u}^{\star}\right|\right\|_{L^{\infty}} \\
& \leq C_{S}\left\|\left|\mathbf{u}_{N}^{\star}-\mathbf{u}_{I}^{\star}\right|\right\|_{H^{1}}+\left\|\left|\mathbf{u}_{I}^{\star}-\mathbf{u}^{\star}\right|\right\|_{L^{\infty}}
\end{aligned}
$$

and utilising

$$
\frac{C_{S} C_{\gamma}}{2 \alpha_{\gamma}} K\left(2 C_{\mathrm{A}}\|\boldsymbol{\xi}\|\right) 2 C_{\mathrm{A}}\|\boldsymbol{\xi}\| \leq \frac{1}{2}
$$

transforms (3.21) into the error results

$$
\left\|\left|\mathbf{u}_{N}^{\star}-\mathbf{u}_{I}^{\star}\right|\right\|_{H^{1}} \leq\left\|\left|\mathbf{u}^{\star}-\mathbf{u}_{I}^{\star}\right|\right\|_{L^{\infty}}+2 \frac{\gamma C_{\gamma}}{1-\beta} \max _{t \geq 0}\left\{e^{-\beta t / 2}\left\|\mathbf{u}^{\star^{\prime}}(t)-\mathbf{u}_{I}^{\star \prime}(t)\right\|\right\}
$$

and

$$
\left\|\left|\mathbf{u}_{N}^{\star}-\mathbf{u}_{I}^{\star}\right|\right\|_{L^{\infty}} \leq\left\|\left|\mathbf{u}^{\star}-\mathbf{u}_{I}^{\star}\right|\right\|_{L^{\infty}}+2 \frac{\gamma C_{S} C_{\gamma}}{1-\beta} \max _{t \geq 0}\left\{e^{-\beta t / 2}\left\|\mathbf{u}^{\star \prime}(t)-\mathbf{u}_{I}^{\star \prime}(t)\right\|\right\}
$$

for each $\beta \in[0,1)$.

Hence our basic error bounds (3.22) depend on the accuracy of polynomial interpolation at Gauss-type points, which is stated in A.19. 
3.4. Superconvergence result. Since we are only interested in the error at $t=0$, i.e., $P^{u}\left[\mathbf{u}^{\star}-\mathbf{u}_{N}^{\star}\right](0)$, any superconvergence property at this point is particularly important. Such a property is well known for many Galerkin-type approximations, and we develop it here for our Laguerre method.

For a given $\mathrm{x} \in \mathcal{E}_{u}$, consider the linear adjoint problem

$$
\mathbf{z}^{\prime}+\frac{1}{\gamma}[\mathrm{A}+\mathrm{B}(t)]^{T} \mathbf{z}=\mathbf{0}
$$

with boundary conditions

$$
\begin{aligned}
Q^{s} \mathbf{z}(0) & =\mathbf{M} \mathbf{x}, \\
\lim _{t \rightarrow \infty} Q^{u} \mathbf{z}(t) & =\mathbf{0},
\end{aligned}
$$

where

$$
\mathrm{B}(t) \equiv \mathrm{J}_{G}\left(e^{\frac{1}{\gamma} \mathrm{A} t} \boldsymbol{\xi}+e^{-t / 2} \mathbf{u}^{\star}\right)
$$

is the Jacobian matrix of $\mathbf{G}$. Just as in the standard constructive existence theorems for the stable manifold of (1.1), we may use the variation of constants approach [1] to write (3.23) in fixed-point form

$$
\mathbf{z}(t)=e^{-\frac{1}{\gamma} \mathrm{A}^{T} t} \mathbf{M} \mathbf{x}-\int_{0}^{t} e^{\frac{1}{\gamma} \mathrm{A}^{T}(\tau-t)} Q^{s} \mathrm{~B}^{T}(\tau) \mathbf{z}(\tau) d \tau+\int_{t}^{\infty} e^{\frac{1}{\gamma} \mathrm{A}^{T}(\tau-t)} Q^{u} \mathrm{~B}^{T}(\tau) \mathbf{z}(\tau) d \tau .
$$

The usual contraction mapping argument then shows that, for $\|\boldsymbol{\xi}\|$ sufficiently small, (3.23) has a unique solution, which we denote by $\widehat{\mathbf{w}}$. For us, the most important point is that this solution inherits the smoothness of $\mathrm{B}$, and all its derivatives are bounded by $\|\mathrm{Mx}\|$; i.e., $\exists C_{\mathrm{B}}>0$ and $\alpha_{\gamma}^{u}>0$ such that

$$
\left\|\widehat{\mathbf{w}}^{(j)}(t)\right\| \leq C_{\mathrm{B}}\|\mathbf{M x}\| e^{-\alpha_{\gamma}^{u} t}, \quad j=0,1,2, \ldots .
$$

( $\alpha_{\gamma}^{u}$ depends on the eigenvalues of $\mathrm{A}_{u}$ and, if all critical eigenvalues are nondefective, we may set $\alpha_{\gamma}^{u} \equiv \lambda^{u} / \gamma$.) Now, if we define

$$
\mathbf{w}(t) \equiv e^{t / 2} \widehat{\mathbf{w}}(t),
$$

the function $\mathbf{w}$ will have the properties that enable us to establish the superconvergence of $\mathbf{u}_{N}^{\star}(0)$, i.e.,

(1) $\mathbf{w}^{\prime}-\left[\frac{1}{2} \mathrm{I}-\frac{1}{\gamma} \mathrm{A}-\frac{1}{\gamma} \mathrm{B}(t)\right]^{T} \mathbf{w}=\mathbf{0}$

(2) $Q^{s} \mathbf{w}(0)=\mathbf{M x}$;

(3) from (3.24) we have $\left\|\left|\mathbf{w}^{(j)}\right|\right\|_{L^{2}}, j=0,1,2, \ldots$, bounded by simple multiples of $\|\mathrm{Mx}\|$.

Thus, through simple integration-by-parts, these first two properties lead to the key formula

$$
-(\mathrm{Mx}) \circ\left[\mathbf{u}_{N}^{\star}-\mathbf{u}^{\star}\right](0)=\left\langle\left(\mathbf{u}_{N}^{\star}-\mathbf{u}_{I}^{\star}\right)^{\prime}-\left[\frac{1}{2} \mathrm{I}+\frac{1}{\gamma} \mathrm{A}+\frac{1}{\gamma} \mathrm{B}(t)\right]\left(\mathbf{u}_{N}^{\star}-\mathbf{u}_{I}^{\star}\right), \mathbf{w}\right\rangle .
$$

Now, if (3.20) and (3.26) are combined, we obtain

$$
\begin{aligned}
&(\mathrm{Mx}) \circ\left[\mathbf{u}_{N}^{\star}-\mathbf{u}^{\star}\right](0)=\langle\left.-\left(\mathbf{u}_{N}^{\star}-\mathbf{u}_{I}^{\star}\right)^{\prime}+\left[\frac{1}{2} \mathbf{I}+\frac{1}{\gamma} \mathrm{A}+\frac{1}{\gamma} \mathrm{B}(t)\right]\left(\mathbf{u}_{N}^{\star}-\mathbf{u}_{I}^{\star}\right), \mathbf{w}-\mathbf{q}\right\rangle \\
&+\frac{1}{\gamma}\left\langle\mathrm{B}(t)\left(\mathbf{u}_{N}^{\star}-\mathbf{u}_{I}^{\star}\right), \mathbf{q}\right\rangle+\left\langle\mathbf{u}_{I}^{\star \prime}-I_{N}\left(\mathbf{u}^{\star \prime}\right), \mathbf{q}\right\rangle \\
&-\frac{1}{\gamma}\left\langle e^{t / 2}\left\{\mathbf{G}\left(e^{\frac{1}{\gamma} \mathrm{A} t} \boldsymbol{\xi}+e^{-t / 2} \mathbf{u}_{N}^{\star}\right)-\mathbf{G}\left(e^{\frac{1}{\gamma} \mathrm{A} t} \boldsymbol{\xi}+e^{-t / 2} \mathbf{u}^{\star}\right)\right\}, \mathbf{q}\right\rangle_{N}
\end{aligned}
$$


for any $\mathbf{q} \in \bar{X}_{N}$. Hence, choosing $\mathbf{q} \equiv \mathbf{w}_{N} \in \bar{X}_{N}$ approximating $\mathbf{w}$, we can split our error into the five terms

$$
(\mathrm{Mx}) \circ\left[\mathbf{u}_{N}^{\star}-\mathbf{u}^{\star}\right](0)=1+2+3+4+5,
$$

where

$$
\begin{aligned}
& 1 \equiv\left\langle-\left(\mathbf{u}_{N}^{\star}-\mathbf{u}_{I}^{\star}\right)^{\prime}+\left[\frac{1}{2} \mathbf{I}+\frac{1}{\gamma} \mathrm{A}+\frac{1}{\gamma} \mathrm{B}(t)\right]\left(\mathbf{u}_{N}^{\star}-\mathbf{u}_{I}^{\star}\right), \mathbf{w}-\mathbf{w}_{N}\right\rangle, \\
& 2 \equiv\left\langle\mathbf{u}_{I}^{\star \prime}-I_{N}\left(\mathbf{u}^{\star \prime}\right), \mathbf{w}_{N}\right\rangle \text {, } \\
& 3 \equiv \frac{1}{\gamma}\left\langle\mathrm{B}(t)\left[\mathbf{u}_{N}^{\star}-\mathbf{u}_{I}^{\star}\right], \mathbf{w}_{N}\right\rangle \\
& -\frac{1}{\gamma}\left\langle P^{s} \mathrm{~B}(t)\left[\mathbf{u}_{N}^{\star}-I_{N}^{G}\left(\mathbf{u}^{\star}\right)\right]+P^{u} \mathrm{~B}(t)\left[\mathbf{u}_{N}^{\star}-I_{N}^{R}\left(\mathbf{u}^{\star}\right)\right], \mathbf{w}_{N}\right\rangle, \\
& 4 \equiv \frac{1}{\gamma}\left\langle P^{s} \mathrm{~B}(t)\left[\mathbf{u}_{N}^{\star}-I_{N}^{G}\left(\mathbf{u}^{\star}\right)\right]+P^{u} \mathrm{~B}(t)\left[\mathbf{u}_{N}^{\star}-I_{N}^{R}\left(\mathbf{u}^{\star}\right)\right], \mathbf{w}_{N}\right\rangle \\
& -\frac{1}{\gamma}\left\langle P^{s} \mathrm{~B}(t)\left[\mathbf{u}_{N}^{\star}-I_{N}^{G}\left(\mathbf{u}^{\star}\right)\right]+P^{u} \mathrm{~B}(t)\left[\mathbf{u}_{N}^{\star}-I_{N}^{R}\left(\mathbf{u}^{\star}\right)\right], \mathbf{w}_{N}\right\rangle_{N}, \\
& 5 \equiv-\frac{1}{\gamma}\left\langle\mathcal{R}(t), \mathbf{w}_{N}\right\rangle_{N} \text {, }
\end{aligned}
$$

with

$$
\begin{aligned}
\mathcal{R}(t) \equiv e^{t / 2}\left\{\mathbf{G}\left(e^{\frac{1}{\gamma} \mathrm{A} t} \boldsymbol{\xi}+e^{-t / 2} \mathbf{u}_{N}^{\star}\right)-\mathbf{G}\left(e^{\frac{1}{\gamma} \mathrm{A} t} \boldsymbol{\xi}+e^{-t / 2} \mathbf{u}^{\star}\right)\right\} \\
-P^{s} \mathrm{~B}(t)\left[\mathbf{u}_{N}^{\star}-I_{N}^{G}\left(\mathbf{u}^{\star}\right)\right]-P^{u} \mathrm{~B}(t)\left[\mathbf{u}_{N}^{\star}-I_{N}^{R}\left(\mathbf{u}^{\star}\right)\right] .
\end{aligned}
$$

Our choice of $\mathbf{w}_{N} \in \bar{X}_{N}$ is based on the fact that we will need both $\left\|\left|\mathbf{w}-\mathbf{w}_{N}\right|\right\|_{L^{2}}$ bounded by $\|\mathbf{M x}\|$ and all derivatives of $\mathbf{w}_{N}$ similarly bounded. Hence, if we take $\mathbf{w}_{N}$ to be the best least-squares fit to $\mathbf{w}$, section A.1 shows how property (3) above leads to these two requirements being satisfied.

All that now remains to be done is the individual bounding of the five terms in $(3.29)$.

- Expression 1 can immediately be bounded by a constant (depending only on $\frac{1}{\gamma},\|\mathrm{A}\|$ and $\left.\max _{t \geq 0}\{\|\mathrm{~B}(t)\|\}\right)$ multiple of

$$
\left\|\left|\mathbf{u}_{N}^{\star}-\mathbf{u}_{I}^{\star}\right|\right\|_{H^{1}}||\left|\mathbf{w}-\mathbf{w}_{N}\right| \|_{L^{2}},
$$

and then we may apply (3.22) and (A.11).

- Expression 2 is small because of the high accuracy of Gauss-type quadratures, and this is apparent if we write

$$
\begin{aligned}
& \left\langle\mathbf{u}_{I}^{\star^{\prime}}-I_{N}\left(\mathbf{u}^{\star^{\prime}}\right), \mathbf{w}_{N}\right\rangle=\left\langle\mathbf{u}_{I}^{\star^{\prime}}-\mathbf{u}^{\star^{\prime}}, \mathbf{w}_{N}\right\rangle+\left\langle\mathbf{u}^{\star^{\prime}}-I_{N}\left(\mathbf{u}^{\star \prime}\right), \mathbf{w}_{N}\right\rangle \\
& =\left\langle\mathbf{u}_{I}^{\star}-\mathbf{u}^{\star}, \mathbf{w}_{N}-\mathbf{w}_{N}^{\prime}\right\rangle+\left\langle\mathbf{u}^{\star \prime}-I_{N}\left(\mathbf{u}^{\star \prime}\right), \mathbf{w}_{N}\right\rangle .
\end{aligned}
$$

Hence, using the notation

$$
\eta_{1} \equiv\left(P^{s} \mathbf{u}^{\star}\right) \circ\left(\mathbf{w}_{N}^{\prime}-\mathbf{w}_{N}\right)+\left(P^{s} \mathbf{u}^{\star \prime}\right) \circ \mathbf{w}_{N}
$$

and

$$
\eta_{2} \equiv\left(P^{u} \mathbf{u}^{\star}\right) \circ\left(\mathbf{w}_{N}^{\prime}-\mathbf{w}_{N}\right)+\left(P^{u} \mathbf{u}^{\star \prime}\right) \circ \mathbf{w}_{N},
$$

we have

$$
2=\int_{0}^{\infty} e^{-t}\left\{\eta_{1}-\phi_{N}^{R} \eta_{1}+\eta_{2}-\widehat{\phi}_{N-1}^{G} \eta_{2}\right\} d t,
$$

which are just quadrature errors and bounded using (A.20) and (A.21). 
- Expression 3 appears because we are using different quadrature rules for the stable and unstable components of our equations. Using the notation $\mathbf{z}(t) \equiv \mathrm{B}^{T}(t) \mathbf{w}_{N}(t), \quad \mathbf{z}^{(1)}(t) \equiv \mathrm{B}^{T}(t) Q^{u} \mathbf{w}_{N}(t) \quad$ and $\quad \mathbf{z}^{(2)}(t) \equiv \mathrm{B}^{T}(t) Q^{s} \mathbf{w}_{N}(t)$,

it can be re-written

$$
\begin{aligned}
& \left\langle\mathrm{B}(t) \mathbf{u}_{I}^{\star}-P^{s} \mathrm{~B}(t) I_{N}^{G}\left(\mathbf{u}^{\star}\right)-P^{u} \mathrm{~B}(t) I_{N}^{R}\left(\mathbf{u}^{\star}\right), \mathbf{w}_{N}\right\rangle \\
& \quad=\left\langle\mathbf{u}_{I}^{\star}-\mathbf{u}^{\star}, \mathbf{z}\right\rangle+\left\langle\mathbf{u}^{\star}-I_{N}^{G}\left(\mathbf{u}^{\star}\right), \mathbf{z}^{(1)}\right\rangle+\left\langle\mathbf{u}^{\star}-I_{N}^{R}\left(\mathbf{u}^{\star}\right), \mathbf{z}^{(2)}\right\rangle .
\end{aligned}
$$

Hence, letting $\mathbf{z}_{N}$ be the best least squares fit to $\mathbf{z}$ from $\bar{X}_{N}, \mathbf{z}_{N}^{(1)}$ the best least squares fit to $\mathbf{z}^{(1)}$ from polynomials of degree $N-1$ or less, and $\mathbf{z}_{N}^{(2)}$ the best least squares fit to $\mathbf{z}^{(2)}$ from polynomials of degree $N$ or less, we have

$$
\begin{aligned}
\left\langle\mathrm{B}(t) \mathbf{u}_{I}^{\star}-P^{s} \mathrm{~B}(t) I_{N}^{G}\left(\mathbf{u}^{\star}\right)-\right. & \left.P^{u} \mathrm{~B}(t) I_{N}^{R}\left(\mathbf{u}^{\star}\right), \mathbf{w}_{N}\right\rangle \\
= & \left\langle\mathbf{u}_{I}^{\star}-\mathbf{u}^{\star}, \mathbf{z}-\mathbf{z}_{N}\right\rangle+\left\langle\mathbf{u}_{I}^{\star}-\mathbf{u}^{\star}, \mathbf{z}_{N}\right\rangle \\
& +\left\langle\mathbf{u}^{\star}-I_{N}^{G}\left(\mathbf{u}^{\star}\right), \mathbf{z}^{(1)}-\mathbf{z}_{N}^{(1)}\right\rangle+\left\langle\mathbf{u}^{\star}-I_{N}^{G}\left(\mathbf{u}^{\star}\right), \mathbf{z}_{N}^{(1)}\right\rangle \\
& +\left\langle\mathbf{u}^{\star}-I_{N}^{R}\left(\mathbf{u}^{\star}\right), \mathbf{z}^{(2)}-\mathbf{z}_{N}^{(2)}\right\rangle+\left\langle\mathbf{u}^{\star}-I_{N}^{R}\left(\mathbf{u}^{\star}\right), \mathbf{z}_{N}^{(2)}\right\rangle .
\end{aligned}
$$

Finally, then, we have 3 equal to $\frac{1}{\gamma}$ multiplying the above right-hand side; the first terms being bounded in sections A.4.1 and A.4.2, while the second terms are quadrature errors and bounded as with expression 2 .

- Expression 4 is basically a quadrature error involving the smooth matrix B; i.e.,

$$
\begin{aligned}
& \left\langle P^{s} \mathrm{~B}(t)\left[\mathbf{u}_{N}^{\star}-I_{N}^{G}\left(\mathbf{u}^{\star}\right)\right]+P^{u} \mathrm{~B}(t)\left[\mathbf{u}_{N}^{\star}-I_{N}^{R}\left(\mathbf{u}^{\star}\right)\right], \mathbf{w}_{N}\right\rangle \\
& \quad=\left\langle\mathbf{u}_{N}^{\star}-I_{N}^{G}\left(\mathbf{u}^{\star}\right), \mathrm{B}^{T}(t) Q^{u} \mathbf{w}_{N}\right\rangle+\left\langle\mathbf{u}_{N}^{\star}-I_{N}^{R}\left(\mathbf{u}^{\star}\right), \mathrm{B}^{T}(t) Q^{s} \mathbf{w}_{N}\right\rangle
\end{aligned}
$$

and

$$
\begin{aligned}
\left\langle P^{s} \mathrm{~B}(t)\left[\mathbf{u}_{N}^{\star}-I_{N}^{G}\left(\mathbf{u}^{\star}\right)\right]+\right. & \left.P^{u} \mathrm{~B}(t)\left[\mathbf{u}_{N}^{\star}-I_{N}^{R}\left(\mathbf{u}^{\star}\right)\right], \mathbf{w}_{N}\right\rangle_{N} \\
= & \sum_{i=1}^{N} w_{i}\left[\mathbf{u}_{N}^{\star}-I_{N}^{G}\left(\mathbf{u}^{\star}\right)\right]\left(t_{i}\right) \circ \mathrm{B}^{T}\left(t_{i}\right) Q^{u} \mathbf{w}_{N}\left(t_{i}\right) \\
& \quad+\sum_{i=0}^{N} \widetilde{w}_{i}\left[\mathbf{u}_{N}^{\star}-I_{N}^{R}\left(\mathbf{u}^{\star}\right)\right]\left(\tilde{t}_{i}\right) \circ \mathrm{B}^{T}\left(\tilde{t}_{i}\right) Q^{s} \mathbf{w}_{N}\left(\tilde{t}_{i}\right) .
\end{aligned}
$$

Hence, using the same notation as with 3 above, we have

$$
4=\frac{1}{\gamma}\left\{\left\langle\mathbf{u}_{N}^{\star}-I_{N}^{G}\left(\mathbf{u}^{\star}\right), \mathbf{z}_{1}-\widehat{I}_{N-1}^{G}\left(\mathbf{z}_{1}\right)\right\rangle+\left\langle\mathbf{u}_{N}^{\star}-I_{N}^{R}\left(\mathbf{u}^{\star}\right), \mathbf{z}_{2}-I_{N}^{R}\left(\mathbf{z}_{2}\right)\right\rangle\right\} .
$$

Our bound for 4 is therefore

$\frac{1}{\gamma}\left\{\left\|\left|\mathbf{u}_{N}^{\star}-I_{N}^{G}\left(\mathbf{u}^{\star}\right)\right|\right\|_{L^{2}}\left\|\left|\mathbf{z}_{1}-\widehat{I}_{N-1}^{G}\left(\mathbf{z}_{1}\right)\right|\right\|_{L^{2}}+\left\|\left|\mathbf{u}_{N}^{\star}-I_{N}^{R}\left(\mathbf{u}^{\star}\right)\right|\right\|_{L^{2}}\left\|\left|\mathbf{z}_{2}-I_{N}^{R}\left(\mathbf{z}_{2}\right)\right|\right\|_{L^{2}}\right\}$,

which is dealt with in section A.4.2.

- Expression 5 is small because it is a Taylor series remainder. To bound it, we must assume that $\mathrm{J}_{G}$ is Lipschitz continuous in a neighbourhood of the origin; i.e., $\exists C_{G}>0$ such that, $\forall \mathbf{x}_{1}, \mathbf{x}_{2}$ sufficiently small,

$$
\left\|\mathbf{G}\left(\mathbf{x}_{2}\right)-\mathbf{G}\left(\mathbf{x}_{1}\right)-\mathrm{J}_{G}\left(\mathbf{x}_{1}\right)\left[\mathbf{x}_{2}-\mathbf{x}_{1}\right]\right\| \leq C_{G}\left\|\mathbf{x}_{2}-\mathbf{x}_{1}\right\|^{2} \text {. }
$$


Then, after applying the Cauchy-Schwarz inequality to 5 to obtain

$$
\begin{aligned}
\left|\left\langle\mathcal{R}(t), \mathbf{w}_{N}\right\rangle_{N}\right| \leq & \left(\sum_{i=1}^{N} w_{i}\left\|P^{s} \mathcal{R}\left(t_{i}\right)\right\|^{2}\right)^{1 / 2}\left(\sum_{i=1}^{N} w_{i}\left\|Q^{u} \mathbf{w}_{N}\left(t_{i}\right)\right\|^{2}\right)^{1 / 2} \\
& +\left(\sum_{i=0}^{N} \widetilde{w}_{i}\left\|P^{u} \mathcal{R}\left(\tilde{t}_{i}\right)\right\|^{2}\right)^{1 / 2}\left(\sum_{i=0}^{N} \widetilde{w}_{i}\left\|Q^{s} \mathbf{w}_{N}\left(\tilde{t}_{i}\right)\right\|^{2}\right)^{1 / 2},
\end{aligned}
$$

we may use $(\dagger)$ to write

$$
\begin{aligned}
\left\|P^{s} \mathcal{R}\left(t_{i}\right)\right\| \leq\left\|P^{s}\right\| C_{G} e^{-t_{i} / 2}\left\|\mathbf{u}^{\star}\left(t_{i}\right)-\mathbf{u}_{N}^{\star}\left(t_{i}\right)\right\|^{2}, & i=1, \ldots, N, \\
\left\|P^{u} \mathcal{R}\left(\tilde{t}_{i}\right)\right\| \leq\left\|P^{u}\right\| C_{G} e^{-\tilde{t}_{i} / 2}\left\|\mathbf{u}^{\star}\left(\tilde{t}_{i}\right)-\mathbf{u}_{N}^{\star}\left(\tilde{t}_{i}\right)\right\|^{2}, & i=0, \ldots, N .
\end{aligned}
$$

Hence, using (A.2e), 5 is bounded by a constant (depending only on the norms of projections) multiple of

$$
\frac{1}{\gamma} C_{G}||\left|\mathbf{u}_{N}^{\star}-\mathbf{u}_{I}^{\star}\right|\left\|_{L^{2}}\right\|\left|\mathbf{u}_{N}^{\star}-\mathbf{u}_{I}^{\star}\right|\left\|_{L^{\infty}}\right\|\left|\mathbf{w}_{N}\right| \|_{L^{2}}
$$

and then we can apply (3.22).

Combining these results we see that:

(1) all terms are of high accuracy, either through products of errors or because of Gauss-type quadrature;

(2) all terms involve derivatives of $\mathbf{w}$ or $\mathbf{w}_{N}$, which are bounded by simple multiples of $\|\mathrm{Mx}\|$.

Thus we can choose $\mathbf{x}=\left[\mathbf{u}_{N}^{\star}-\mathbf{u}^{\star}\right](0)$ in (3.28) and obtain

$$
\left\|\left[\mathbf{u}^{\star}-\mathbf{u}_{N}^{\star}\right](0)\right\| \leq \sqrt{\|\mathrm{M}\|\left\|\mathrm{M}^{-1}\right\|} \times \text { "superconvergence". }
$$

\section{IMPLEMENTATION}

For an efficient implementation of (2.10) or (2.11) we must choose and construct matrices

$$
\mathrm{U} \in \mathbb{R}^{m \times p^{s}} \quad \text { and } \quad \mathrm{V} \in \mathbb{R}^{m \times p^{u}}
$$

whose columns form bases for $\mathcal{E}_{s}$ and $\mathcal{E}_{u}$, respectively. It is preferable for these bases to be orthonormal, i.e., Schur vectors [12]; if available, however, a well-conditioned eigenvector basis may be more convenient. We then have available the matrices

$$
\mathrm{A}_{-} \in \mathbb{R}^{p^{s} \times p^{s}} \quad \text { and } \quad \mathrm{A}_{+} \in \mathbb{R}^{p^{u} \times p^{u}},
$$

which denote the restrictions of $\mathrm{A}$ to $\mathcal{E}_{s}$ and $\mathcal{E}_{u}$, respectively, with respect to the two bases above. Next we need to define

$$
\mathrm{W}_{-} \in \mathbb{R}^{p^{s} \times m} \quad \text { and } \quad \mathrm{W}_{+} \in \mathbb{R}^{p^{u} \times m}
$$

by

$$
\mathbf{x}=\mathbf{U} \mathbf{u}+V_{\mathbf{v}} \quad \Longrightarrow \quad W_{-} \mathbf{x}=\mathbf{u} \text { and } W_{+} \mathbf{x}=\mathbf{v}
$$

i.e.,

$$
\left[\begin{array}{l}
\mathrm{W}_{-} \\
\mathrm{W}_{+}
\end{array}\right]=\left[\begin{array}{ll}
\mathrm{U} & \mathrm{V}
\end{array}\right]^{-1}
$$

Finally, we require $\boldsymbol{\xi}_{i} \in \mathbb{R}^{p^{s}}, i=1, \ldots, N$, and $\tilde{\boldsymbol{\xi}}_{i} \in \mathbb{R}^{p^{s}}, i=0, \ldots, N$, such that

$$
\mathrm{U} \boldsymbol{\xi}_{i} \equiv e^{\frac{1}{\gamma} \mathrm{A} t_{i}} \boldsymbol{\xi}, \quad i=1, \ldots, N, \quad \text { or } \quad \boldsymbol{\xi}_{i}=e^{\frac{1}{\gamma} \mathrm{A}_{-} t_{i}} \mathrm{~W}_{-} \boldsymbol{\xi}, \quad i=1, \ldots, N,
$$


and

$$
\mathrm{U} \tilde{\boldsymbol{\xi}}_{i} \equiv e^{\frac{1}{\gamma} \mathrm{A} \tilde{t}_{i}} \boldsymbol{\xi}, \quad i=0, \ldots, N, \quad \text { or } \quad \tilde{\boldsymbol{\xi}}_{i}=e^{\frac{1}{\gamma} \mathrm{A}-\tilde{t}_{i}} \mathrm{~W}_{-} \boldsymbol{\xi}, \quad i=0, \ldots, N
$$

Since $A_{-}$will be in Schur form or better, the matrix exponentials can be easily computed [12].

We can now form the practical implementation of (2.10) by expressing our discrete solution in the form

$$
\mathbf{u}_{N}\left(t_{i}\right) \equiv \mathbf{U} \mathbf{u}_{i}+\mathrm{V}_{i}, \quad i=1, \ldots, N,
$$

and

$$
\mathbf{u}_{N}\left(\tilde{t}_{i}\right) \equiv \mathrm{U} \tilde{\mathbf{u}}_{i}+\mathrm{V}_{i}, \quad i=0, \ldots, N
$$

where $\mathbf{u}_{i} / \tilde{\mathbf{u}}_{i} \in \mathbb{R}^{p^{s}}$ and $\mathbf{v}_{i} / \tilde{\mathbf{v}}_{i} \in \mathbb{R}^{p^{u}}$ with $\tilde{\mathbf{u}}_{0}=\mathbf{0}$. Then our equations corresponding to $(2.10 \mathrm{~b})-(2.10 \mathrm{c})$ are

$$
\begin{aligned}
& \sum_{j=1}^{N} d_{i j} \mathbf{u}_{j}-\left[\frac{1}{2} \mathbf{I}+\frac{1}{\gamma} \mathrm{A}_{-}\right] \mathbf{u}_{i} \\
& \quad=\frac{1}{\gamma} e^{t_{i} / 2} \mathbf{W}_{-} \mathbf{G}\left(\mathbf{U} \boldsymbol{\xi}_{i}+e^{-t_{i} / 2}\left[\mathbf{U} \mathbf{u}_{i}+\mathbf{V}_{i}\right]\right), \quad i=1, \ldots, N
\end{aligned}
$$

and

$$
\begin{aligned}
& \sum_{j=0}^{N} \tilde{d}_{i j} \tilde{\mathbf{v}}_{j}-\left[\frac{1}{2} \mathrm{I}+\frac{1}{\gamma} \mathrm{A}_{+}\right] \tilde{\mathbf{v}}_{i} \\
& \quad=\frac{1}{\gamma} e^{\tilde{t}_{i} / 2} \mathbf{W}_{+} \mathbf{G}\left(\mathbf{U} \tilde{\boldsymbol{\xi}}_{i}+e^{-\tilde{t}_{i} / 2}\left[\mathbf{U} \tilde{\mathbf{u}}_{i}+\mathbf{V} \tilde{\mathbf{v}}_{i}\right]\right), \quad i=0, \ldots, N .
\end{aligned}
$$

Finally, we change variables in (4.4) to

$$
\hat{\mathbf{u}}_{i} \equiv\left[\sqrt{w_{i}} / \sqrt{t_{i}}\right] \mathbf{u}_{i} \quad i=1, \ldots, N, \quad \text { and } \quad \hat{\mathbf{v}}_{i} \equiv \sqrt{\widetilde{w}_{i}} \tilde{\mathbf{v}}_{i}, \quad i=0, \ldots, N,
$$

so that, by making use of (A.6) and (A.9), we have only these $N p^{s}+(N+1) p^{u}$ unknowns; i.e.,

$$
\begin{aligned}
& \sum_{j=1}^{N} d_{i j}^{\prime} \hat{\mathbf{u}}_{j}-\left[\frac{1}{2} \mathrm{I}+\frac{1}{\gamma} \mathrm{A}_{-}\right] \hat{\mathbf{u}}_{i} \\
& \quad=\frac{1}{\gamma} \frac{\sqrt{w_{i}^{\prime}}}{\sqrt{t_{i}}} \mathbf{W}_{-} \mathbf{G}\left(\mathrm{U} \boldsymbol{\xi}_{i}+\frac{1}{\sqrt{w_{i}^{\prime}}}\left[\mathrm{U} \hat{\mathbf{u}}_{i}+\mathrm{V}(\mathcal{T} \hat{\mathbf{v}})_{i}\right]\right), i=1, \ldots, N
\end{aligned}
$$

and

$$
\begin{aligned}
& \sum_{j=0}^{N} \tilde{d}_{i j}^{\prime} \hat{\mathbf{v}}_{j}-\left[\frac{1}{2} \mathrm{I}+\frac{1}{\gamma} \mathrm{A}_{+}\right] \hat{\mathbf{v}}_{i} \\
& \quad=\frac{1}{\gamma} \sqrt{\widetilde{w}_{i}^{\prime}} \mathrm{W}_{+} \mathbf{G}\left(\mathrm{U} \tilde{\boldsymbol{\xi}}_{i}+\frac{1}{\sqrt{\widetilde{w}_{i}^{\prime}}}\left[\mathrm{U}(\tilde{\mathcal{T}} \hat{\mathbf{u}})_{i}+\mathrm{V} \hat{\mathbf{v}}_{i}\right]\right), i=0, \ldots, N .
\end{aligned}
$$


Thus the mappings $\mathcal{T}$

\begin{tabular}{|l|ll|ll}
\hline $\mathcal{T}$ & INPUT $:$ & $\hat{\mathbf{v}} \equiv \hat{\mathbf{v}}_{0}, \ldots, \hat{\mathbf{v}}_{N}$ & OUTPUT $:$ & $\mathcal{T} \hat{\mathbf{v}} \equiv(\mathcal{T} \hat{\mathbf{v}})_{1}, \ldots,(\mathcal{T} \hat{\mathbf{v}})_{N}$ \\
\hline
\end{tabular}

a) Apply $\widetilde{\mathbf{S}}$ to each component of $\hat{\mathbf{v}}_{0}, \ldots, \hat{\mathbf{v}}_{N}$ to obtain $\sum_{k=0}^{N} \mathbf{a}_{k} L_{k}(t)$.

b) Drop $\mathbf{a}_{N}$, since $L_{N}(t)$ is zero at $t_{1}, \ldots t_{N}$.

c) Apply $\mathrm{S}^{T}$ to each component of $\mathbf{a}_{0}, \ldots, \mathbf{a}_{N-1}$ to obtain $(\mathcal{T} \hat{\mathbf{v}})_{1}, \ldots,(\mathcal{T} \hat{\mathbf{v}})_{N}$.

and $\widetilde{\mathcal{T}}$

\begin{tabular}{|l|l|ll}
\hline$\widetilde{\mathcal{T}}$ & INPUT $: \hat{\mathbf{u}} \equiv \hat{\mathbf{u}}_{1}, \ldots, \hat{\mathbf{u}}_{N}$ & OUTPUT $:$ & $\widetilde{\mathcal{T}} \hat{\mathbf{u}} \equiv(\widetilde{\mathcal{T}} \hat{\mathbf{u}})_{0}, \ldots,(\widetilde{\mathcal{T}} \hat{\mathbf{u}})_{N}$ \\
\hline
\end{tabular}

a) Apply $\mathbf{S}$ to each component of $\hat{\mathbf{u}}_{1}, \ldots, \hat{\mathbf{u}}_{N}$ to obtain $\sum_{k=0}^{N-1} \mathbf{a}_{k} L_{k}(t)$.

b) Set $\mathbf{a}_{N}=-\sum_{k=0}^{N-1} \mathbf{a}_{k}$, thus imposing the boundary condition at $t=0$.

c) Apply $\widetilde{\mathbf{S}}^{T}$ to each component of $\mathbf{a}_{0}, \ldots, \mathbf{a}_{N}$ to obtain $(\widetilde{\mathcal{T}} \hat{\mathbf{u}})_{0}, \ldots,(\widetilde{\mathcal{T}} \hat{\mathbf{u}})_{N}$.

efficiently convert nodal values of polynomials between $t_{1}, \ldots, t_{N}$ and $\tilde{t}_{0}, \ldots, \tilde{t}_{N}$, using (A.6). An additional key point here is that the condition of $\mathrm{D}_{N}^{\prime}$ and $\widetilde{\mathrm{D}}_{N}^{\prime}$, unlike that of their unscaled counterparts $\mathrm{D}_{N}$ and $\widetilde{\mathrm{D}}_{N}$, does not deteriorate with $N$ [17. Note that, from (A.4b), the unstable component of the point on the stable manifold is given by $\sqrt{N+1} \vee \hat{\mathbf{v}}_{0}$.

Similarly, we can now form the practical implementation of (2.11) by setting

$$
\mathbf{a}_{i}^{s} \equiv \mathrm{U} \hat{\mathbf{a}}_{i}^{s}, \quad \mathbf{g}_{i}^{s} \equiv \mathrm{U} \hat{\mathbf{g}}_{i}^{s}, \quad \mathbf{a}_{i}^{u} \equiv \mathrm{V} \hat{\mathbf{a}}_{i}^{u}, \quad \mathbf{g}_{i}^{u} \equiv \mathrm{V} \hat{\mathbf{g}}_{i}^{u},
$$

where $\hat{\mathbf{a}}_{i}^{s} / \hat{\mathbf{g}}_{i}^{s} \in \mathbb{R}^{p^{s}}$ and $\hat{\mathbf{a}}_{i}^{u} / \hat{\mathbf{g}}_{i}^{u} \in \mathbb{R}^{p^{u}}$. Then our equations corresponding to (2.11) are

$$
\begin{gathered}
\sum_{k=0}^{N} \hat{\mathbf{a}}_{k}^{s}=\mathbf{0}, \\
\sum_{k=0}^{i-1} \hat{\mathbf{a}}_{k}^{s}+\left[\frac{1}{2} \mathbf{I}-\frac{1}{\gamma} \mathrm{A}_{-}\right] \hat{\mathbf{a}}_{i}^{s}=\frac{1}{\gamma} \hat{\mathbf{g}}_{i}^{s}, \quad i=0, \ldots, N-1, \\
-\sum_{k=i+1}^{N} \hat{\mathbf{a}}_{k}^{u}-\left[\frac{1}{2} \mathbf{I}+\frac{1}{\gamma} \mathrm{A}_{+}\right] \hat{\mathbf{a}}_{i}^{u}=\frac{1}{\gamma} \hat{\mathbf{g}}_{i}^{u}, \quad i=0, \ldots, N,
\end{gathered}
$$

where

$$
\begin{gathered}
\hat{\mathrm{g}}^{s}=\mathcal{S} \hat{\mathbf{c}}^{s} \quad \text { with } \hat{\mathbf{c}}^{s} \equiv \hat{\mathbf{c}}_{1}^{s}, \ldots, \hat{\mathbf{c}}_{N}^{s}, \\
\hat{\mathbf{c}}_{i}^{s} \equiv \sqrt{w_{i}^{\prime}} \mathrm{W}_{-} \mathbf{G}\left(\mathrm{U} \boldsymbol{\xi}_{i}+\frac{1}{\sqrt{w_{i}^{\prime}}}\left[\mathcal{S}^{T} \mathbf{a}\right]_{i}\right)
\end{gathered}
$$

and

$$
\begin{gathered}
\hat{\mathbf{g}}^{u}=\widetilde{\mathcal{S}} \hat{\mathbf{c}}^{u} \quad \text { with } \hat{\mathbf{c}}^{u} \equiv \hat{\mathbf{c}}_{0}^{u}, \ldots, \hat{\mathbf{c}}_{N}^{u}, \\
\hat{\mathbf{c}}_{i}^{u} \equiv \sqrt{\widetilde{w}_{i}^{\prime}} \mathrm{W}_{+} \mathbf{G}\left(\mathrm{U} \tilde{\boldsymbol{\xi}}_{i}+\frac{1}{\sqrt{\widetilde{w}_{i}^{\prime}}}\left[\widetilde{\mathcal{S}}^{T} \mathbf{a}\right]_{i}\right)
\end{gathered}
$$


with $\mathbf{a} \equiv \mathrm{U} \hat{\mathbf{a}}_{i}^{s}+\mathrm{V} \hat{\mathbf{a}}_{i}^{u}, i=0, \ldots, N$. Here, $\mathcal{S}, \mathcal{S}^{T}, \widetilde{\mathcal{S}}$ and $\widetilde{\mathcal{S}}^{T}$ are just the vector forms of the matrices $\mathrm{S}, \mathrm{S}^{T}, \widetilde{\mathrm{S}}$ and $\widetilde{\mathrm{S}}^{T}$ in (A.7) so that

$$
\begin{aligned}
& \mathbf{g}_{i-1}^{s} \equiv\left[\mathcal{S} \hat{\mathbf{c}}^{s}\right]_{i}=\sum_{j=1}^{N} s_{i j} \hat{\mathbf{c}}_{j}^{s}, \quad i=1, \ldots, N \\
& {\left[\mathcal{S}^{T} \mathbf{a}\right]_{i}=\sum_{j=1}^{N} s_{j i} \mathbf{a}_{j-1}, \quad i=1, \ldots, N,}
\end{aligned}
$$

and

$$
\begin{aligned}
& \mathbf{g}_{i}^{u} \equiv\left[\widetilde{\mathcal{S}} \hat{\mathbf{c}}^{u}\right]_{i}=\sum_{j=0}^{N} \tilde{s}_{i j} \hat{\mathbf{c}}_{j}^{u}, \quad i=0, \ldots, N, \\
& {\left[\widetilde{\mathcal{S}}^{T} \mathbf{a}\right]_{i}=\sum_{j=0}^{N} \tilde{s}_{j i} \mathbf{a}_{j}, \quad i=0, \ldots, N . }
\end{aligned}
$$

4.1. Conclusion. It is now time to make a final comparison between the collocation formulation (4.5) and the expansion formulation (4.7), which we have so far developed in tandem. It is natural to solve either of these nonlinear systems using the fixed point approach of subsection 3.2, i.e., solving a succession of linear problems with the right-hand side of (4.5) or (4.7) known at each iteration. It is then clear that (4.7) has the enormous advantage that it may be solved in the manner of subsection 3.1, i.e.,

- (4.7b), (4.7a) is a "lower-triangular" set of equations, which can be solved by the forward-recurrence

$$
\begin{aligned}
\hat{\mathbf{a}}_{i}^{s} & =\left[\frac{\gamma}{2} \mathbf{l}-\mathrm{A}_{-}\right]^{-1}\left(\hat{\mathbf{g}}_{i}^{s}-\gamma \sum_{k=0}^{i-1} \hat{\mathbf{a}}_{k}^{s}\right), \quad i=0, \ldots, N-1, \\
\hat{\mathbf{a}}_{N}^{s} & =-\sum_{k=0}^{N-1} \hat{\mathbf{a}}_{k}^{s} ;
\end{aligned}
$$

- (4.7c) is an "upper-triangular" set of equations, which can be solved by the backward-recurrence

$$
\hat{\mathbf{a}}_{i}^{u}=-\left[\frac{\gamma}{2} \mathbf{I}+\mathrm{A}_{+}\right]^{-1}\left(\gamma \sum_{k=i+1}^{N} \hat{\mathbf{a}}_{k}^{u}+\hat{\mathbf{g}}_{i}^{u}\right), \quad i=N, \ldots, 0
$$

On the other hand, with (4.5) it is necessary to solve an $N p^{s}$ system to obtain $\hat{\mathbf{u}}_{1}, \ldots, \hat{\mathbf{u}}_{N}$ and an $(N+1) p^{u}$ system to obtain $\hat{\mathbf{v}}_{0}, \ldots, \hat{\mathbf{v}}_{N}$. Hence our numerical results in the next section are based on (4.7). We have been willing to derive the collocation framework, however, since the particular structure of $\mathrm{D}_{N}^{\prime}$ and $\widetilde{\mathrm{D}}_{N}^{\prime}$ may lead to special efficient solution methods being developed. 
Finally, we note that the analogue of (4.7)-(4.8) for the implementation of (2.13)$(2.14)$ is

$$
\begin{gathered}
\sum_{k=0}^{N} \hat{\mathbf{a}}_{k}^{s}=\tilde{\boldsymbol{\xi}}_{0}, \\
-\tilde{\boldsymbol{\xi}}_{0}+\sum_{k=0}^{i-1} \hat{\mathbf{a}}_{k}^{s}+\left[\frac{1}{2} \mathbf{I}-\frac{1}{\gamma} \mathrm{A}_{-}\right] \hat{\mathbf{a}}_{i}^{s}=\frac{1}{\gamma} \hat{\mathbf{g}}_{i}^{s}, \quad i=0, \ldots, N-1, \\
-\sum_{k=i+1}^{N} \hat{\mathbf{a}}_{k}^{u}-\left[\frac{1}{2} \mathbf{I}+\frac{1}{\gamma} \mathrm{A}_{+}\right] \hat{\mathbf{a}}_{i}^{u}=\frac{1}{\gamma} \hat{\mathbf{g}}_{i}^{u}, \quad i=0, \ldots, N,
\end{gathered}
$$

where

$$
\begin{gathered}
\hat{\mathbf{g}}^{s}=\mathcal{S} \hat{\mathbf{c}}^{s} \quad \text { with } \hat{\mathbf{c}}^{s} \equiv \hat{\mathbf{c}}_{1}^{s}, \ldots, \hat{\mathbf{c}}_{N}^{s} \\
\hat{\mathbf{c}}_{i}^{s} \equiv \sqrt{w_{i}^{\prime}} \mathrm{W}_{-} \mathbf{G}\left(\frac{1}{\sqrt{w_{i}^{\prime}}}\left[\mathcal{S}^{T} \mathbf{a}\right]_{i}\right)
\end{gathered}
$$

and

$$
\begin{aligned}
& \hat{\mathbf{g}}^{u}=\widetilde{\mathcal{S}} \hat{\mathbf{c}}^{u} \quad \text { with } \hat{\mathbf{c}}^{u} \equiv \hat{\mathbf{c}}_{0}^{u}, \ldots, \hat{\mathbf{c}}_{N}^{u}, \\
& \hat{\mathbf{c}}_{i}^{u} \equiv \sqrt{\widetilde{w}_{i}^{\prime}} \mathrm{W}_{+} \mathbf{G}\left(\frac{1}{\sqrt{\widetilde{w}_{i}^{\prime}}}\left[\widetilde{\mathcal{S}}^{T} \mathbf{a}\right]_{i}\right) .
\end{aligned}
$$

\section{Numerical RESUlts}

Now we illustrate the above algorithms on two simple well-known examples.

\subsection{The Lorenz equations.}

$$
\begin{aligned}
& \dot{x}=a(y-x), \\
& \dot{y}=b x-y-x z, \\
& \dot{z}=x y-c z .
\end{aligned}
$$

We use the parameter values $(a, b, c)=\left(10,14, \frac{8}{3}\right)$ for which the stationary solution at the origin is hyperbolic. The Jacobian matrix there has real eigenvalues

$$
-c, \quad-\frac{1}{2}\left[(a+1) \pm \sqrt{(a+1)^{2}+4 a(b-1)}\right],
$$

two of which are negative. We take

$$
\boldsymbol{\xi}=\delta\left(\phi_{1}+\phi_{2}\right)
$$

(where $\phi_{i}$ are the normalised eigenvectors corresponding to the two negative eigenvalues) and $\delta=4$.

Figures 5.1 and 5.2 show the error in the unstable component of the stable manifold for algorithms (2.11) and (2.13), respectively, with various values of $\gamma$. The exponential convergence with respect to $N$ is clear in both cases. In Figure 5.1 the results seem to improve as $\gamma$ increases up to $\gamma=35$, but then the error for $\gamma=40$ is somewhat larger. Since there is no $z^{2}$ term in the Lorenz equations, the exponential decay rate of our solution is $e^{-20.83 t}$. This is being approximated by a polynomial multiplied by $e^{-\frac{\gamma}{2} t}$, and thus a value of $\gamma \approx 41.65$ is to be expected. 


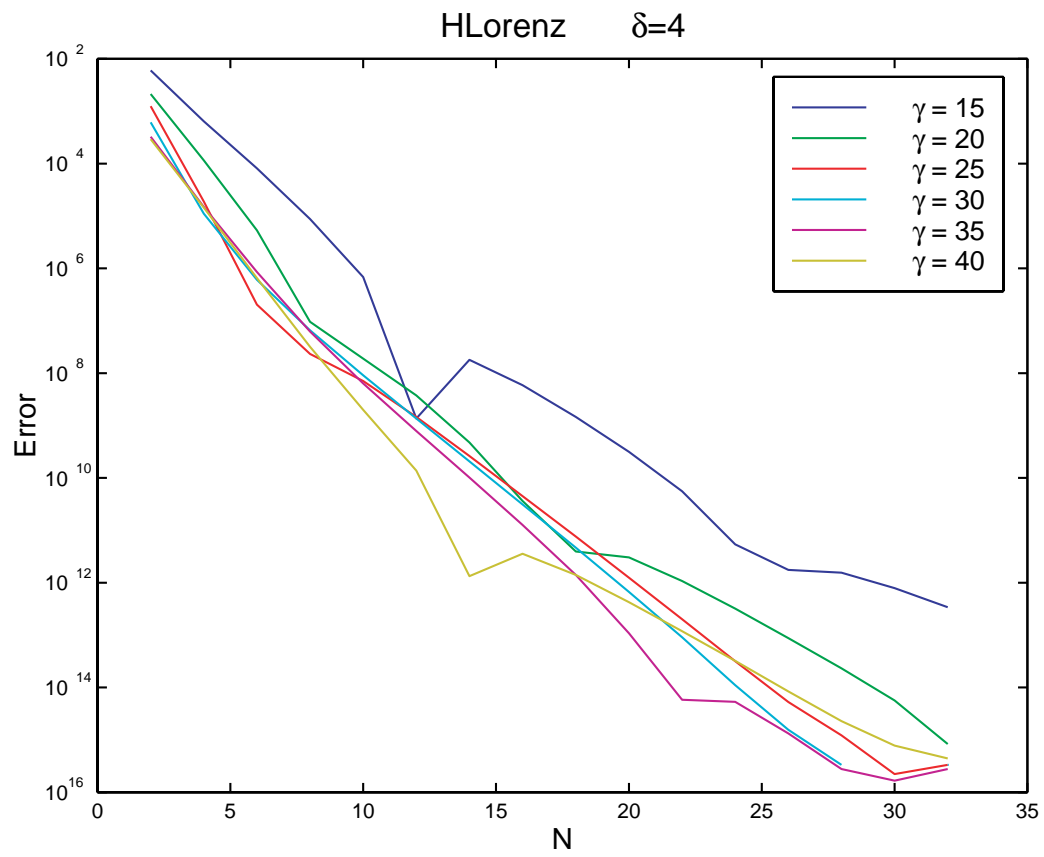

FIGURE 5.1. Algorithm with homogeneous initial condition

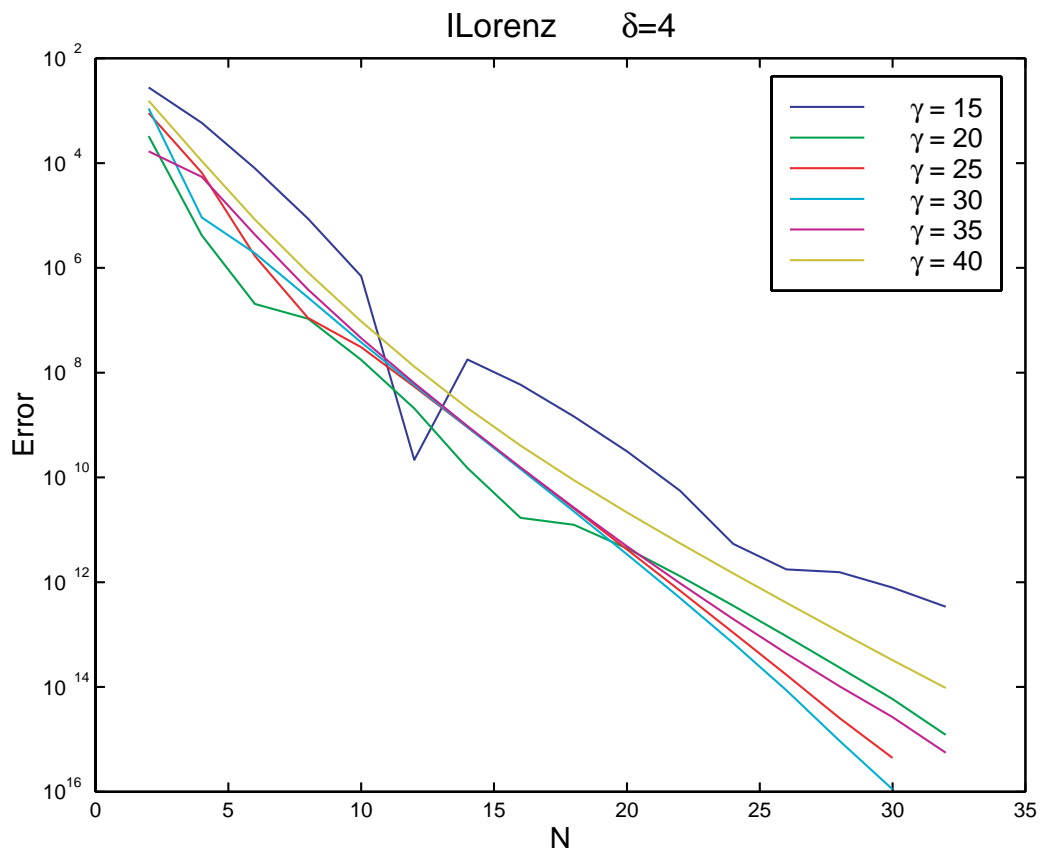

FigURE 5.2. Algorithm with inhomogeneous initial condition 
In contrast, however, if the $\mathbf{w}-\mathbf{w}_{N}$ term in (3.29) is critical, the best value of $\gamma$ would be $\gamma \approx 14.32$. Thus we are not surprised that Figure 5.1 seems to indicate an optimal value of $\gamma$ somewhat less than 40. In Figure 5.2 the results seem to improve as $\gamma$ increases up to $\gamma=30$, but $\gamma=40$ definitely gives a larger error. Although there are now components of the solution that decay like $e^{-\frac{8}{3} t}$, these are in the stable subspace and do not directly affect the accuracy of our manifold approximation. Nevertheless, it is clear that there is now a stronger influence toward smaller $\gamma$ and also that the errors in Figure 5.2 are somewhat larger than those in Figure 5.1.

\subsection{Chua's electronic circuit.}

$$
\begin{aligned}
& \dot{x}=a\left(y+\frac{1}{6}\left(x-x^{3}\right)\right), \\
& \dot{y}=x-y+z, \\
& \dot{z}=-b y .
\end{aligned}
$$

We use the parameter values $(a, b)=(4,5)$ for which the stationary solution at the origin is again hyperbolic. The Jacobian matrix there has one positive eigenvalue and a pair of complex conjugate eigenvalues with negative real part, i.e., approximately 1.32 and $-0.83 \pm 1.36 i$. We take

$$
\boldsymbol{\xi}=\delta\left(\phi_{R}+\phi_{I}\right)
$$

(where $\phi_{R}$ and $\phi_{I}$ are the real and imaginary parts of the complex eigenvector) and $\delta=1.5$.

Figures 5.3 and 5.4 show the error in the unstable component of the stable manifold for algorithms (2.11) and (2.13), respectively, with various values of $\gamma$.

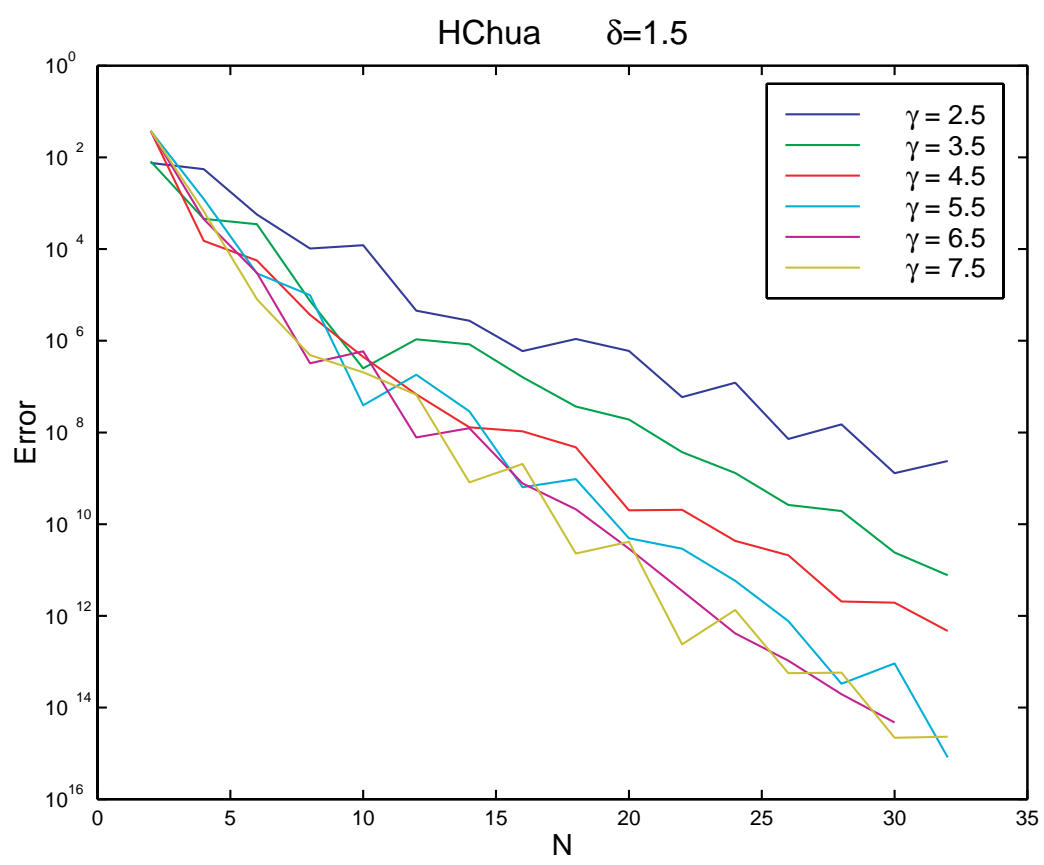

FIGURE 5.3. Algorithm with homogeneous initial condition 


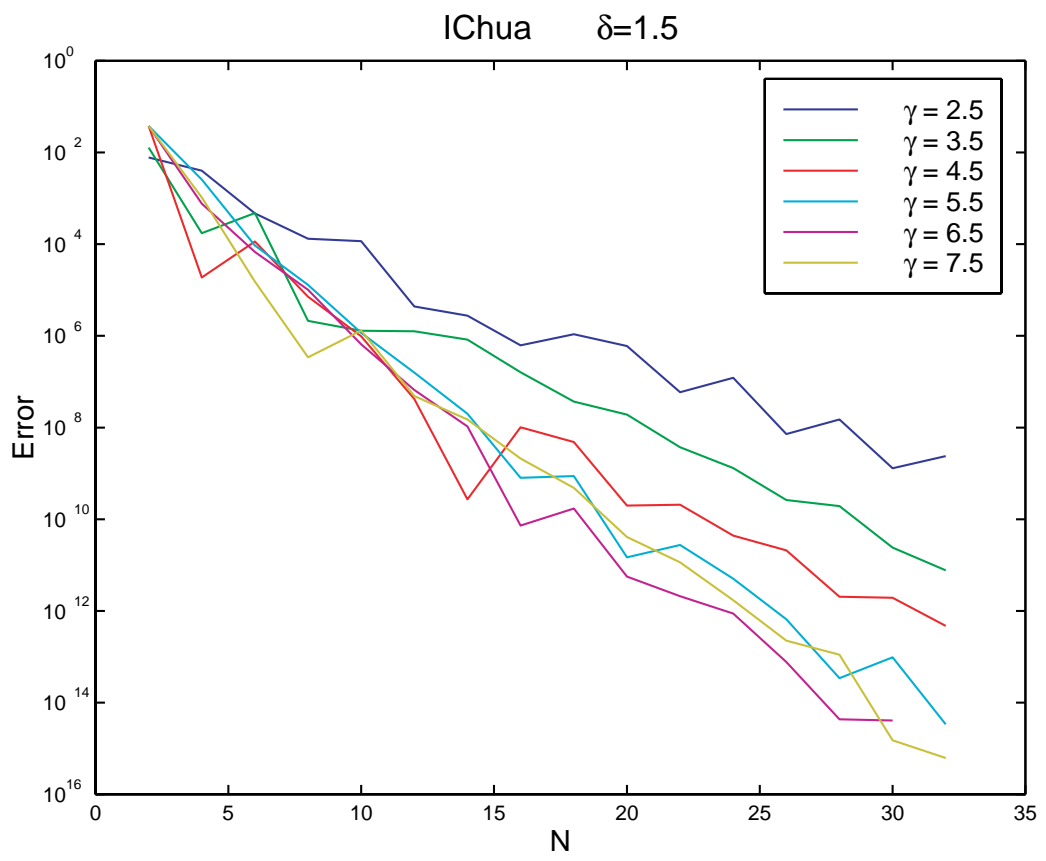

FIGURE 5.4. Algorithm with inhomogeneous initial condition

Again the exponential convergence with respect to $N$ is clear in both cases. In Figure 5.3 the results seem to improve as $\gamma$ increases up to $\gamma=6.5$, and then the error for $\gamma=7.5$ is about the same. Since there is no quadratic term in Chua's equation, the exponential decay rate of our solution is $e^{-2.48 t}$; thus a good value of $\gamma$ is expected to be $\gamma \approx 4.97$. It is not obvious, however, why the error decreases for larger values of $\gamma$. The influence of the $\mathbf{w}-\mathbf{w}_{N}$ term in (3.29) does not seem important here, since this would indicate a value of $\gamma \approx 2.64$. In Figure 5.4 the error for $\gamma=7.5$ seems definitely worse than that for $\gamma=6.5$. Apart from this, neither algorithm seems significantly preferable to the other.

5.3. Choice of $\gamma$. In the light of the above numerical results, a simple formula for the optimal value of $\gamma$ is unlikely to exist. We suggest, nevertheless, that setting $\frac{\gamma}{2}$ equal to the decay rate of the unstable component of the solution is always a good choice. Further improvement in the choice of $\gamma$ must be empirical and problem dependent. We also have a slight preference for the algorithm with homogeneous initial conditions.

\section{Concluding Remarks}

6.1. Homoclinic and heteroclinic orbits. Our main reason for studying Laguerre approximation of stable manifolds in this paper is so that we can eventually develop a fully spectral approximation of connecting orbits between stationary solutions [3], 9], [19. Here we very briefly describe the basic idea. We replace (1.1) with the parameter-dependent problem

$$
\mathbf{v}^{\prime}=\mathbf{F}(\mathbf{v}, \lambda), \quad \mathbf{F}: \mathbb{R}^{m} \times \mathbb{R}^{q} \mapsto \mathbb{R}^{m}
$$


and assume that $\mathbf{v}_{ \pm}: \mathbb{R}^{q} \mapsto \mathbb{R}^{m}$ are two (possibly identical) hyperbolic stationary solution curves of (6.1), i.e., $\mathbf{F}\left(\mathbf{v}_{ \pm}(\lambda), \lambda\right)=\mathbf{0}$ and the Jacobian matrices $\mathrm{J}_{F}\left(\mathbf{v}_{ \pm}(\lambda), \lambda\right)$ have no eigenvalues on the imaginary axis. (The number of free parameters, $q$, must be such that the sum of the dimensions of the stable manifold of $\mathbf{v}_{+}(\lambda)$ and the unstable manifold of $\mathbf{v}_{-}(\lambda)$ equals $m+1-q[3$.) Then, to approximate a connecting orbit between $\mathbf{v}_{-}(\lambda)$ and $\mathbf{v}_{+}(\lambda)$, we solve the three coupled equations

$$
\begin{array}{rlrl}
\mathbf{v}_{u}^{\prime}(t) & =\mathbf{F}\left(\mathbf{v}_{u}(t), \lambda\right), & t \in(-\infty, 0), \\
\mathbf{v}_{c}^{\prime}(\tau) & =T \mathbf{F}\left(\mathbf{v}_{c}(\tau), \lambda\right), & & \tau \in(-1,1), \\
\mathbf{v}_{s}^{\prime}(t) & =\mathbf{F}\left(\mathbf{v}_{s}(t), \lambda\right), & t \in(0, \infty),
\end{array}
$$

with

$$
\mathbf{v}_{u}(0)=\mathbf{v}_{c}(-1) \quad \text { and } \quad \mathbf{v}_{c}(1)=\mathbf{v}_{s}(0) .
$$

I.e., $\mathbf{v}_{u}(t)$ is a trajectory lying in the unstable manifold of $\mathbf{v}_{-}(\lambda), \mathbf{v}_{s}(t)$ is a trajectory lying in the stable manifold of $\mathbf{v}_{+}(\lambda)$, while $\mathbf{v}_{c}(\tau)$ is an orbit (parametrised by scaled time; cf. [9]) connecting these two manifolds. Thus our unknowns to be determined are $\mathbf{v}_{u}, \mathbf{v}_{c}, \mathbf{v}_{s}, \lambda$ and $T$. To complete a well-posed problem, we must also specify what proportions of the full connecting orbit are contained in (6.2a) and $(6.2 \mathrm{c})$. One possibility is to choose $\varepsilon_{ \pm}>0$ sufficiently small and to insist that

$$
\int_{-\infty}^{0}\left\|\mathbf{F}\left(\mathbf{v}_{u}, \lambda\right)\right\| d t=\varepsilon_{-} \quad \text { and } \quad \int_{0}^{\infty}\left\|\mathbf{F}\left(\mathbf{v}_{s}, \lambda\right)\right\| d t=\varepsilon_{+}
$$

cf. [2], [20]. Of course, when discretising (6.2) we would use Laguerre approximation for $(6.2 \mathrm{a})$ and $(6.2 \mathrm{c})$, and either Chebyshev or Legendre approximation for $(6.2 \mathrm{~b})$. Further details and results for such spectral algorithms will be given elsewhere: here we just show a graph of results for the model problem in [4], i.e., $m=2, q=1$ and

$$
\begin{aligned}
& \dot{x}=y, \\
& \dot{y}=\lambda-2 y-x^{2}+x y .
\end{aligned}
$$

The identical stationary solutions are

$$
\mathbf{v}_{-}(\lambda) \equiv \mathbf{v}_{+}(\lambda)=\left(\begin{array}{c}
-\sqrt{\lambda} \\
0
\end{array}\right)
$$

and a homoclinic orbit exists for $\lambda \approx 6.5$. We use $\varepsilon_{ \pm}=1$ and apply Laguerre approximation with $\gamma=3$ to $(6.2 \mathrm{a})$ and with $\gamma=20$ to $(6.2 \mathrm{c})$.

Figure 6.1 shows the error in the value of $\lambda$ for the homoclinic orbit computed from different orders of polynomial approximation. Note that for fixed order Laguerre approximation, the rate of convergence is exponential in $N$ until the Laguerre error becomes dominant. (Zero-order Laguerre corresponds to approximation of the stable/unstable manifolds by the corresponding stable/unstable subspaces, which is the standard technique in $[\underline{3}, 9]$.)

We aim to produce a Matlab implementation of this approximation scheme, with (6.2b) replaced by the arclength parametrisation of [19], i.e.,

$$
\mathbf{v}_{c}^{\prime}(\sigma)=L \frac{\mathbf{F}\left(\mathbf{v}_{c}(\sigma), \lambda\right)}{\left\|\mathbf{F}\left(\mathbf{v}_{c}(\sigma), \lambda\right)\right\|}, \quad \sigma \in(-1,1)
$$




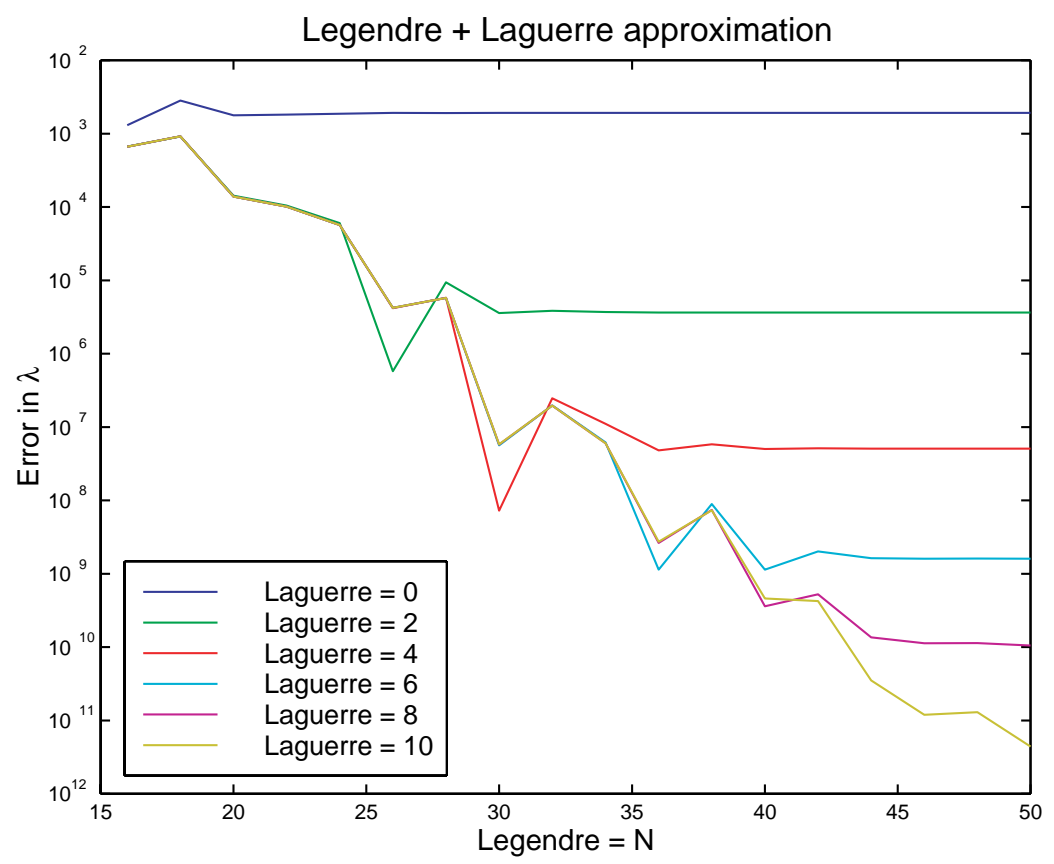

Figure 6.1. Approximation of homoclinic orbit

Comparison of this approach with the popular HOMCONT package [6], 8] will be presented elsewhere.

6.2. Strongly stable manifolds. We can also extend the approximation scheme in this paper to strongly stable manifolds of $\rho$-pseudohyperbolic stationary solutions of (1.1) [23], 24]; i.e., $\rho<0$ is such that none of the eigenvalues of $A$ in (1.2) has real part equal to $\rho$. Instead of the changes of variable at the beginning of section 2 , we apply the transformations

i) $t \mapsto t / \gamma, \quad \gamma>0$

ii) $\mathbf{u}(t)=e^{\sigma t} \mathbf{v}(t), \quad \sigma=\frac{1}{2}-\frac{\rho}{\gamma}$

to (1.1) and obtain

$$
\mathbf{u}^{\prime}-\left[\sigma \mathrm{I}+\frac{1}{\gamma} \mathrm{A}\right] \mathbf{u}=\frac{e^{\sigma t}}{\gamma} \mathbf{G}\left(e^{-\sigma t} \mathbf{u}\right)
$$

(Note that, for $\rho=0$, (6.5) corresponds to the equation being approximated by (2.5).) This enables us to calculate any trajectory of (1.1) such that

$$
e^{-\rho t} \mathbf{v}(t) \rightarrow 0 \quad \text { as } t \rightarrow \infty
$$

and, as in the present paper, $\gamma$ can be tuned to optimize the approximation of this strongly stable manifold. In particular, if our stationary solution is nonhyperbolic, we can approximate points on the stable manifold by choosing $|\rho|$ sufficiently small. Of course, by replacing $\mathbf{F}$ by $-\mathbf{F}$, a similar extension holds for unstable manifolds. 
Appendix. LAguerre polynomials, QuAdrature AND APPROXIMATION

In this section, for convenience, we collect together some well-known results to which we wish to refer; cf. [7], [10], [17], [18], [22]. The famous Laguerre polynomials $L_{N}, \quad N=0,1,2, \ldots$, have the following properties:

(1) Differential equation

$$
t L_{N}^{\prime \prime}(t)+[1-t] L_{N}^{\prime}(t)+N L_{N}(t)=0 .
$$

(2) Orthogonality

$$
\int_{0}^{\infty} e^{-t} L_{N_{1}}(t) L_{N_{2}}(t) d t=\delta_{N_{1}, N_{2}}
$$

(3) Recurrence relation

$$
\begin{aligned}
N L_{N}(t)= & {[2 N-1-t] L_{N-1}(t)-[N-1] L_{N-2}(t), } \\
& L_{0}(t)=1, \quad L_{1}(t)=1-t .
\end{aligned}
$$

(As is standard, the polynomials have been scaled so that $L_{N}(0)=1$.) It then follows that, if

$$
p_{N}(t)=\sum_{k=0}^{N} a_{k} L_{k}(t) \text { and } p_{N}^{\prime}(t)=\sum_{k=0}^{N-1} b_{k} L_{k}(t)
$$

we have
(A.1a) $a_{0}=p_{N}(0)+b_{0}$,
$a_{k}=b_{k}-b_{k-1}, \quad k=1, \ldots, N-1$,
$a_{N}=-b_{N-1}$

or

$$
b_{k}=-\sum_{j=k+1}^{N} a_{j} \equiv-p_{N}(0)+\sum_{j=0}^{k} a_{j}
$$

In addition, the orthogonality property implies

$$
\int_{0}^{\infty} e^{-t}\left[p_{N}(t)\right]^{2} d t \equiv \sum_{k=0}^{N} a_{k}^{2} \quad \text { and } \quad \int_{0}^{\infty} e^{-t}\left[p_{N}^{\prime}(t)\right]^{2} d t \equiv \sum_{k=0}^{N-1} b_{k}^{2} .
$$

A.1. Quadrature rules. In this paper we are particularly concerned with the Gauss-Laguerre quadrature rule

$$
\int_{0}^{\infty} e^{-t} f(t) d t \approx \sum_{j=1}^{N} w_{j} f\left(t_{j}\right)
$$

and the Gauss-Laguerre-Radau rule

$$
\int_{0}^{\infty} e^{-t} f(t) d t \approx \sum_{j=0}^{N} \widetilde{w}_{j} f\left(\tilde{t}_{j}\right)
$$

where $\tilde{t}_{0}=0$. (The former is exact if $f$ is a polynomial of degree $2 N-1$ or less, while the latter is exact if $f$ is a polynomial of degree $2 N$ or less; for example,

$$
p_{N-1}(t)=\sum_{k=0}^{N-1} a_{k} L_{k}(t) \Longrightarrow \sum_{j=1}^{N} w_{j}\left[p_{N-1}\left(t_{j}\right)\right]^{2}=\sum_{k=0}^{N-1} a_{k}^{2}
$$


and

$$
p_{N}(t)=\sum_{k=0}^{N} a_{k} L_{k}(t) \Longrightarrow \sum_{j=0}^{N} \widetilde{w}_{j}\left[p_{N}\left(\tilde{t}_{j}\right)\right]^{2}=\sum_{k=0}^{N} a_{k}^{2} .
$$

In addition, we shall need the important result

$$
\sum_{j=1}^{N} w_{j}\left[p_{N}\left(t_{j}\right)\right]^{2} \leq \int_{0}^{\infty} e^{-t}\left[p_{N}(t)\right]^{2} d t,
$$

which is based on an expansion in Laguerre polynomials.) The classical formulae for these nodes and weights are

$$
\begin{aligned}
& t_{1}, \ldots, t_{N} \text { are the roots of } L_{N} \\
& \tilde{t}_{1}, \ldots, \tilde{t}_{N} \text { are the roots of } L_{N+1}^{\prime},
\end{aligned}
$$

and

$$
\begin{aligned}
& w_{j}=\frac{1}{t_{j}}\left[L_{N}^{\prime}\left(t_{j}\right)\right]^{-2}, \quad j=1, \ldots, N, \\
& \widetilde{w}_{j}=\frac{1}{N+1}\left[L_{N+1}\left(\tilde{t}_{j}\right)\right]^{-2}, \quad j=0, \ldots, N .
\end{aligned}
$$

These quadrature weights decay exponentially; more precisely,

$$
\left.\begin{array}{l}
\sum_{j=1}^{N} w_{j} e^{\beta t_{j}} \\
\sum_{j=0}^{N} \widetilde{w}_{j} e^{\beta \tilde{t}_{j}}
\end{array}\right\} \leq \frac{1}{1-\beta}
$$

for $\beta \in[0,1$ ). For $\beta=1$ there is very slow growth with $N$ (cf. 22]) and it is convenient to define

$$
w_{j}^{\prime} \equiv w_{j} e^{t_{j}} \quad \text { and } \quad \widetilde{w}_{j}^{\prime} \equiv \widetilde{w}_{j} e^{\tilde{t}_{j}}
$$

A.2. Tridiagonal eigenproblems. Since [13, however, these values have usually been obtained from the solution of eigenproblems; see also 11]. Thus $t_{1}, \ldots, t_{N}$ are the eigenvalues of the $N \times N$ symmetric tridiagonal matrix

$$
\mathrm{T}_{N}=\left(\begin{array}{cccccc}
1 & -1 & & & & \\
-1 & 3 & -2 & & & \\
& -2 & 5 & \ddots & & \\
& & \ddots & \ddots & \ddots & \\
& & & \ddots & \ddots & -(N-1) \\
& & & & -(N-1) & 2 N-1
\end{array}\right)
$$

and, if $\mathrm{S}_{N}$ denotes the corresponding orthogonal matrix of eigenvectors,

$$
w_{j}=s_{1 j}^{2}=\left(\mathrm{S}_{N}\right)_{1 j}^{2}, \quad j=1, \ldots, N .
$$


Similarly, $\tilde{t}_{0}, \ldots, \tilde{t}_{N}$ are the eigenvalues of the $(N+1) \times(N+1)$ symmetric tridiagonal matrix

$$
\widetilde{\mathrm{T}}_{N}=\left(\begin{array}{cccccc}
1 & -1 & & & & \\
-1 & 3 & -2 & & & \\
& -2 & \ddots & \ddots & & \\
& & \ddots & \ddots & \ddots & \\
& & & \ddots & 2 N-1 & -N \\
& & & & -N & N
\end{array}\right)
$$

and, if $\widetilde{\mathrm{S}}_{N}$ denotes the corresponding orthogonal matrix of eigenvectors (with row/column numbering $0, \ldots, N)$,

$$
\widetilde{w}_{j}=\tilde{s}_{0 j}^{2}=\left(\widetilde{\mathrm{S}}_{N}\right)_{0 j}^{2}, \quad j=0, \ldots, N .
$$

If they are chosen so that the first element in each column is positive, the eigenvector matrices $S_{N}$ and $\widetilde{S}_{N}$ also define the mappings between expansion coefficients and scaled nodal values for polynomials. Thus, if

$$
p_{N}(t) \equiv \sum_{k=0}^{N} a_{k} L_{k}(t) \quad \text { and } \quad c_{j} \equiv \sqrt{w_{j}} p_{N}\left(t_{j}\right), \quad j=1, \ldots, N,
$$

then

$$
\mathbf{a}=\mathrm{S}_{N} \mathbf{c} \text { and } \mathbf{c}=\mathrm{S}_{N}^{T} \mathbf{a},
$$

where $\mathbf{a} \equiv\left(\mathbf{a}_{0}, \ldots, \mathbf{a}_{N-1}\right)^{T}$; cf. (A.3a). Similarly, if

$$
p_{N}(t) \equiv \sum_{k=0}^{N} a_{k} L_{k}(t) \quad \text { and } \quad c_{j} \equiv \sqrt{\widetilde{w}_{j}} p_{N}\left(\tilde{t}_{j}\right), \quad j=0, \ldots, N,
$$

then

$$
\mathbf{a}=\widetilde{\mathrm{S}}_{N} \mathbf{c} \text { and } \mathbf{c}=\widetilde{\mathrm{S}}_{N}^{T} \mathbf{a} .
$$

Since a fast Laguerre transform has not been discovered (although optimism was expressed in [17]), these mappings provide the most convenient transformations.

A.3. Derivative matrices. The nodes and weights also enable us to define simple linear mappings between scaled nodal values of a polynomial and the corresponding scaled nodal values of its derivative. Thus, if $p_{N}(0)=0$ and

$$
\begin{aligned}
c_{j} & \equiv\left[\sqrt{w_{j}} / \sqrt{t_{j}}\right] p_{N}\left(t_{j}\right), \quad j=1, \ldots, N, \\
\hat{c}_{j} & \equiv\left[\sqrt{w_{j}} / \sqrt{t_{j}}\right] p_{N}^{\prime}\left(t_{j}\right), \quad
\end{aligned}
$$

then

$$
\hat{\mathbf{c}}=\mathrm{D}_{N}^{\prime} \mathbf{c},
$$

where

$$
\left(\mathrm{D}_{N}^{\prime}\right)_{i j} \equiv d_{i j}^{\prime}= \begin{cases}1 /\left(t_{i}-t_{j}\right) & i \neq j \\ \left(1+t_{i}\right) /\left(2 t_{i}\right) & i=j .\end{cases}
$$


Similarly, if

$$
\begin{aligned}
& c_{j} \equiv \sqrt{\widetilde{w}_{j}} p_{N}\left(\tilde{t}_{j}\right), \\
& \hat{c}_{j} \equiv \sqrt{\widetilde{w}_{j}} p_{N}^{\prime}\left(\tilde{t}_{j}\right), \quad j=0, \ldots, N,
\end{aligned}
$$

then

$$
\hat{\mathbf{c}}=\widetilde{\mathrm{D}}_{N}^{\prime} \mathbf{c}
$$

where

$$
\left(\widetilde{\mathrm{D}}_{N}^{\prime}\right)_{i j} \equiv \tilde{d}_{i j}^{\prime}= \begin{cases}1 /\left(\tilde{t}_{i}-\tilde{t}_{j}\right) & i \neq j, \\ 1 / 2 & i=j \neq 0 \\ -N / 2 & i=j=0 .\end{cases}
$$

A.4. Approximation errors. Finally, again for reference, we state some of the approximation error bounds derived in [17] and that are relevant to the present paper. $\mathbb{P}_{N}$ is used to denote the space of polynomials of degree $N$ or less.

A.4.1. Least squares. $\pi_{N} u \in \mathbb{P}_{N}$ is defined by

$$
\int_{0}^{\infty} e^{-t}\left[\pi_{N} u\right](t) p_{N}(t) d t=\int_{0}^{\infty} e^{-t} u(t) p_{N}(t) d t \quad \forall p_{N} \in \mathbb{P}_{N} .
$$

It is shown in [17] that, for any $\varepsilon>0$ and positive integer $p$, the Laguerre coefficients of $u$ decrease spectrally, i.e.,

$$
\left|\int_{0}^{\infty} e^{-t} u(t) L_{k}(t) d t\right| \leq C(p, \varepsilon) k^{-p / 2}\|u\|_{p, 1-\varepsilon}
$$

where

$$
\|z\|_{p, \beta} \equiv\left\{\sum_{j=0}^{p} \int_{0}^{\infty} e^{-\beta t}\left|z^{(j)}(t)\right|^{2} d t\right\}^{1 / 2} .
$$

(Here and later $C(p, \varepsilon)$ is a constant whose exact form can be derived from [17. For us, it is only important that:

(1) $C(p, \varepsilon)$ grows with $p$ like $C^{p / 2}$, where $C$ is an absolute constant, and hence is eventually dominated by a term like $N^{-p / 2}$;

(2) $C(p, \varepsilon)$ grows as $\varepsilon$ decreases and is unbounded as $\varepsilon \rightarrow 0$.)

From (A.10), it then easily follows that

$$
\left\|u-\pi_{N} u\right\|_{0,1} \leq C(p, \varepsilon) N^{-p / 2}\|u\|_{p, 1-\varepsilon}
$$

and

$$
\left\|u^{\prime}-\left(\pi_{N} u\right)^{\prime}\right\|_{0,1} \leq C(p, \varepsilon) N^{1-p / 2}\|u\|_{p, 1-\varepsilon},
$$

the latter bound utilising the identity

$$
\left(\pi_{N} u\right)^{\prime}=\pi_{N}\left(u^{\prime}\right)+a_{N}^{(1)} L_{N+1}^{\prime},
$$

where

$$
a_{N}^{(m)} \equiv \int_{0}^{\infty} e^{-t} u^{(m)}(t) L_{N}(t) d t .
$$


In fact, provided that $u$ is sufficiently smooth, all derivatives of $\pi_{N} u$ may be bounded by repeatedly applying this identity to obtain

$$
\left(\pi_{N} u\right)^{(s)}=\pi_{N}\left(u^{(s)}\right)+\sum_{k=1}^{s} a_{N}^{(s-k+1)} L_{N+1}^{(k)}, \quad s=1, \ldots, N .
$$

A little algebra, using $L_{j}^{\prime}=-\sum_{i=0}^{j-1} L_{i}$, shows that

$$
L_{N+1}^{(k)}=\sum_{j=0}^{N+1-k} \alpha_{j} L_{j} \quad \text { with } \quad \sum_{j=0}^{N+1-k}\left|\alpha_{j}\right|=\left(\begin{array}{c}
N+1 \\
k
\end{array}\right)
$$

and hence

$$
\left\|L_{N+1}^{(k)}\right\|_{0,1} \leq 2^{N}, \quad k=1, \ldots, N .
$$

Inserting (A.10) and (A.13) in (A.12) then gives

$$
\left\|\pi_{N} u\right\|_{N, 1} \leq\|u\|_{N, 1}+C(2 N, \varepsilon) \frac{2^{N} N^{-N / 2}}{1-1 / N}\|u\|_{2 N, 1-\varepsilon} .
$$

A.4.2. Interpolation. Let $\phi_{N} u$ denote either of the following interpolation operators:

$$
\begin{aligned}
& \phi_{N}^{G} u \in \mathbb{P}_{N} \quad \text { and } \quad\left(\phi_{N}^{G} u\right)\left(t_{i}\right)=u\left(t_{i}\right), \quad i=0, \ldots, N, \\
& \phi_{N}^{R} u \in \mathbb{P}_{N} \quad \text { and } \quad\left(\phi_{N}^{R} u\right)\left(\tilde{t}_{i}\right)=u\left(\tilde{t}_{i}\right), \quad i=0, \ldots, N,
\end{aligned}
$$

where $t_{0} \equiv 0$. Then, using Sobolev embedding and inverse inequalities, the interpolation error is $N^{1 / 2}$ worse than the least-squares error (A.11); i.e., for any $\varepsilon>0$ and positive integer $p$

$$
\begin{aligned}
\left\|u-\phi_{N} u\right\|_{0,1} & \leq C(p, \varepsilon) N^{(1-p) / 2}\|u\|_{p, 1-\varepsilon} \\
\left\|u^{\prime}-\left(\phi_{N} u\right)^{\prime}\right\|_{0,1} & \leq C(p, \varepsilon) N^{(3-p) / 2}\|u\|_{p, 1-\varepsilon} .
\end{aligned}
$$

Similarly, if $\widehat{\phi}_{N-1}^{G} u$ denotes the interpolation operator defined by

$$
\widehat{\phi}_{N-1}^{G} u \in \mathbb{P}_{N-1} \quad \text { and } \quad\left(\widehat{\phi}_{N-1}^{G} u\right)\left(t_{i}\right)=u\left(t_{i}\right), \quad i=1, \ldots, N,
$$

then, for any $\varepsilon>0$ and positive integer $p$,

$$
\begin{aligned}
\left\|u-\widehat{\phi}_{N-1}^{G} u\right\|_{0,1} & \leq C(p, \varepsilon)(N-1)^{(1-p) / 2}\|u\|_{p, 1-\varepsilon}, \\
\left\|u^{\prime}-\left(\widehat{\phi}_{N-1}^{G} u\right)^{\prime}\right\|_{0,1} & \leq C(p, \varepsilon)(N-1)^{(3-p) / 2}\|u\|_{p, 1-\varepsilon} .
\end{aligned}
$$

(We note that [17 only analyses interpolation at Gauss-Radau points, but the arguments are similar in the other two cases.) Analogous weighted maximum-norm results are also possible, e.g.,

$$
\begin{aligned}
\left\|u-\phi_{N} u\right\|_{0, \sigma, \infty} & \leq C(p, \sigma, \varepsilon) N^{5 / 4-p / 2}\|u\|_{p, \sigma-\varepsilon}, \\
\left\|u^{\prime}-\left(\phi_{N} u\right)^{\prime}\right\|_{0, \sigma, \infty} & \leq C(p, \sigma, \varepsilon) N^{9 / 4-p / 2}\|u\|_{p, \sigma-\varepsilon},
\end{aligned}
$$

where

$$
\|z\|_{p, \beta, \infty} \equiv \max _{\substack{t \geq 0 \\ 0 \leq k \leq p}}\left\{e^{-\beta t}\left|z^{(k)}(t)\right|\right\}
$$


A.4.3. Quadrature. The error in Gauss or Gauss-Radau quadrature can be written in various ways. For us, however, it is appropriate to combine the least-squares error with Sobolev embedding and inverse inequalities. Thus for Gauss-Radau quadrature, i.e., $\phi_{N}^{R}$ defined by (A.15b), we have

$$
\begin{aligned}
\int_{0}^{\infty} & e^{-t}\left\{u(t)-\left[\phi_{N}^{R} u\right](t)\right\} d t \\
& =\int_{0}^{\infty} e^{-t}\left\{u(t)-\left[\pi_{2 N} u\right](t)\right\} d t-\int_{0}^{\infty} e^{-t} \phi_{N}^{R}\left[u-\pi_{2 N} u\right](t) d t
\end{aligned}
$$

and hence

$$
\left|\int_{0}^{\infty} e^{-t}\left\{u(t)-\left[\phi_{N}^{R} u\right](t)\right\} d t\right| \leq C(2 N, \varepsilon)(2 N)^{(1-p) / 2}\|u\|_{p, 1-\varepsilon} .
$$

Similarly, for Gauss quadrature, i.e., $\widehat{\phi}_{N-1}^{G}$ defined by (A.17), we have

$$
\begin{aligned}
\int_{0}^{\infty} & e^{-t}\left\{u(t)-\left[\widehat{\phi}_{N-1}^{G} u\right](t)\right\} d t \\
& =\int_{0}^{\infty} e^{-t}\left\{u(t)-\left[\pi_{2 N-1} u\right](t)\right\} d t-\int_{0}^{\infty} e^{-t} \widehat{\phi}_{N-1}^{G}\left[u-\pi_{2 N-1} u\right](t) d t
\end{aligned}
$$

and hence

$$
\left|\int_{0}^{\infty} e^{-t}\left\{u(t)-\left[\widehat{\phi}_{N-1}^{G} u\right](t)\right\} d t\right| \leq C(2 N-1, \varepsilon)(2 N-1)^{(1-p) / 2}\|u\|_{p, 1-\varepsilon} .
$$

\section{REFERENCES}

1. H. Amann, Ordinary Differential Equations, Studies in Mathematics 13, de Gruyter, 1990. MR 91e:34001

2. Z. Bashir-Ali, J.R. Cash and G. Moore, Numerical experiments with algorithms for connecting orbits, in preparation.

3. W-J. Beyn, The numerical computation of connecting orbits in dynamical systems., I.M.A. Jour. Num. Anal. 9 (1990), 379-405. MR 91i:65146

4. W-J. Beyn, Numerical methods for dynamical systems, Advances In Numerical Analysis (W. Light, ed.), Clarendon Press, Oxford, 1991, pp. 175-236. MR 92f:65005

5. C. Canuto, M.Y. Hussaini, A. Quarteroni and T.A. Zang, Spectral Methods in Fluid Mechanics, Springer, New York, 1987. MR 89m:76004

6. A.R. Champneys, Y.A. Kuznetsov and B. Sandstede, A numerical toolbox for homoclinic bifurcation analysis, Int. J. Bif. \& Chaos 6 (1996), 867-887. MR 98b:65094

7. P.J. Davis and P. Rabinowitz, Methods of Numerical Integration, Academic Press, New York, 1975. MR 56:7119

8. E.J. Doedel, A.R. Champneys, T.F. Fairgrieve, Y.A. Kuznetsov, B. Sandstede \& X.J. Wang, AUTO 97: Continuation And Bifurcation Software for Ordinary Differential Equations (with HomCont), Technical Report, California Institute Of Technology, 1998.

9. M.J. Friedman and E.J. Doedel, Numerical computation and continuation of invariant manifolds connecting fixed points, SIAM J. Numer. Anal. 28 (1991), 789-808. MR 98e:34058

10. D. Funaro, Computational aspects of pseudospectral Laguerre approximations, Applied Numerical Mathematics 6 (1989/90), 447-457. MR 91m:65074

11. W. Gautschi, Orthogonal polynomials: applications and computation, Acta Numerica 5 (A. Iserles, ed.), Cambridge University Press, 1996, pp. 45-119. MR 99j:65019

12. G.H. Golub and C.F. van Loan, Matrix Computations, John Hopkins, Baltimore, 1989. MR 90d: 65055

13. G.H. Golub and J.H. Welsch, Calculation of Gauss quadrature rules, Math. Comp. 23 (1969), 221-230. MR 39:6513

14. J. Guckenheimer and P.H. Holmes, Nonlinear Oscillations, Dynamical Systems, and Bifurcations of Vector Fields, Applied Mathematical Sciences Vol. 42, Springer, 1983. MR 85f:58002 
15. M.W. Hirsch and S. Smale, Differential Equations. Dynamical Systems and Linear Algebra, Academic Press, San Diego, 1974. MR 58:6484

16. Y. Liu, L. Liu and T. Tang, The numerical computation of connecting orbits in dynamical systems: a rational spectral approach, J. Comput. Phys. 111 (1994), 373-380. MR 95a:65109

17. Y. Maday, B. Pernaud-Thomas and H. Vandeven, Reappraisal of Laguerre type spectral methods, Rech. Aerosp. (1985-86), 13-35. MR 88b:65135

18. C. Mavriplis, Laguerre polynomials for infinite-domain spectral elements, J. Comput. Phys. 80 (1989), 480-488. MR 90f:65228

19. G. Moore, Computation and parametrisation of periodic and connecting orbits., I.M.A. Jour. Num. Anal. 15 (1995), 245-263. MR 96a:34087

20. G. Moore, Geometric methods for computing invariant manifolds, Applied Numerical Mathematics 17 (1995), 319-331. MR 96g:65082

21. G. Moore and E. Hubert, Algorithms for constructing stable manifolds of stationary solutions, I.M.A. Jour. Num. Anal. 19 (1999), 375-424. MR 2000f:65066

22. P. Rabinowitz and G. Weiss, Tables of abscissas and weights for numerical evaluation of integrals of the form $\int_{0}^{\infty} e^{-x} x^{n} f(x) d x$, Math. Tables Aids Comp. 13 (1959), 285-294. MR 21:6713

23. D. Ruelle, Elements of Differentiable Dynamics and Bifurcation Theory, Academic Press, San Diego, 1989. MR 90f:58048

24. M. Shub, Global Stability of Dynamical Systems, Springer, New York, 1987. MR 87m:58086

Department of Mathematics, Imperial College, Queen's Gate, London SW7 2BZ EngLAND

E-mail address: g.moore@ic.ac.uk 